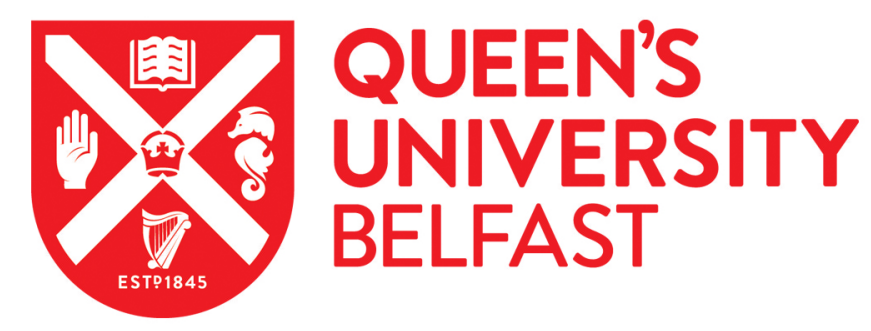

\title{
Mechanochemical Synthesis of Pharmaceutical Cocrystal Suspensions via Hot Melt Extrusion: Feasibility Studies and Physicochemical Characterisation
}

Li, S., Yu, T., Tian, Y., McCoy, C. P., Jones, D. S., \& Andrews, G. P. (2016). Mechanochemical Synthesis of Pharmaceutical Cocrystal Suspensions via Hot Melt Extrusion: Feasibility Studies and Physicochemical Characterisation. Molecular Pharmaceutics, 13(9), 3054-3068.

https://doi.org/10.1021/acs.molpharmaceut.6b00134

\section{Published in:}

Molecular Pharmaceutics

\section{Document Version:}

Peer reviewed version

Queen's University Belfast - Research Portal:

Link to publication record in Queen's University Belfast Research Portal

\section{Publisher rights}

Copyright 2016 American Chemical Society.

\section{General rights}

Copyright for the publications made accessible via the Queen's University Belfast Research Portal is retained by the author(s) and / or other copyright owners and it is a condition of accessing these publications that users recognise and abide by the legal requirements associated with these rights.

Take down policy

The Research Portal is Queen's institutional repository that provides access to Queen's research output. Every effort has been made to ensure that content in the Research Portal does not infringe any person's rights, or applicable UK laws. If you discover content in the Research Portal that you believe breaches copyright or violates any law, please contact openaccess@qub.ac.uk. 
1 Mechanochemical Synthesis of Pharmaceutical Cocrystal Suspensions via Hot Melt

2 Extrusion: Feasibility Studies and Physicochemical Characterisation

3

4 Shu Li, Tao Yu, Yiwei Tian, Colin P. McCoy, David S. Jones and Gavin P. Andrews*,

5 Pharmaceutical Engineering Group, School of Pharmacy, Medical Biology Centre,

6 Queen's University, Belfast BT9, Northern Ireland

7

8

$9{ }^{*}$ Correspondence to: Gavin P. Andrews (Telephone: +44-2890-97-2646; E-mail:

10 g.andrews@qub.ac.uk)

11

12

13

14 Running Title: Cocrystals via Mechanochemistry

15 


\section{Abstract:}

17 Engineered Cocrystals offer an alternative solid drug form with tailored 18 physicochemical properties. Interestingly, although cocrystals provide many new possibilities they also present new challenges, particularly in regard to their design and large-scale manufacture. Current literature has primarily focused on the preparation and characterization of novel cocrystals typically containing only

22 the drug and coformer, leaving the subsequent formulation less explored. In this 23 paper we propose, for the first time, the use of hot melt extrusion for the 24 mechanochemical synthesis of pharmaceutical cocrystals in the presence of a meltable binder. In this approach, we examine excipients that are amenable to

26 hot melt extrusion, forming a suspension of cocrystal particulates embedded in a 27 pharmaceutical matrix. Using ibuprofen and isonicotinamide as a model cocrystal reagent pair, formulations extruded with a small molecular matrix carrier (xylitol) were examined to be intimate mixtures wherein the newly formed cocrystal particulates were physically suspended in a matrix. With respect to formulations extruded using polymeric carriers (Soluplus ${ }^{\circledR}$ and 32 Eudragit ${ }^{\circledR}$ EPO, respectively), however, there was no evidence within PXRD 33 patterns of either crystalline ibuprofen or the cocrystal. Importantly, it was 34 established in this study that an appropriate carrier for a cocrystal reagent pair during HME processing should satisfy certain criteria including limited interaction with parent reagents and cocrystal product, processing temperature

37 sufficiently lower than the onset of cocrystal $\mathrm{T}_{\mathrm{m}}$, low melt viscosity and rapid solidification upon cooling. 
40 Pharmaceutical material science is fundamental in the design and development

41 of new and improved drug delivery platforms. In particular, crystal engineering has brought the possibility of designing new drug complexes (cocrystals) that provide an opportunity to modify the properties of the parent drug. Possible improvements include solid-state properties, aqueous solubility, dissolution rate and bioavailability ${ }^{1-3}$, the latter being particularly important for BCS Class II drugs. Undoubtedly, over the last decade, the use of crystal engineering to optimise the physical properties of drugs has gained significant attention ${ }^{4,5}$. This may be attributed to the fact that the effective delivery of drugs to a patient in a safe and cost-effective manner is significantly influenced by the physicochemical properties of drug in the solid state. Moreover, cocrystals manufactured via crystal engineering offer an alternative solid drug form with tailored physicochemical properties and represent a significant opportunity to generate intellectual property. Interestingly, although cocrystals provide many new possibilities they also present new challenges, particularly in regard to their design and large-scale manufacture.

Pharmaceutical cocrystals are multi-component molecular complexes consisting of a drug and a cocrystal former (coformer) in a well-defined stoichiometry, formed mainly via hydrogen bonding, halogen bonds and/or $\pi-\pi$ stacking supramolecular interactions ${ }^{4-6}$. The wide range of coformer properties and interactions in solid and solution phase (depending upon manufacturing method) provides an opportunity to alter physicochemical properties. It has been previously reported that successful cocrystallization requires complimentary functional groups on drug and coformer, typical examples including carboxylic acids and amides ${ }^{7}$.

Cocrystals are traditionally manufactured using traditional solvent evaporation $^{8,9}$. However, more recently there has been a strong and increasing demand for clean and environmentally friendly processes that focus on green methods of conducting chemical reactions in the absence of solvents. Of particular relevance within the pharmaceutical arena has been the recent interest in, and success of, grinding methods, for pharmaceutical cocrystallisation via mechanochemical reactions ${ }^{10-13}$. This has been driven by the 
fact that pharmaceutical cocrystal synthesis is largely due to the formation of supramolecular interactions that can be broken and reformed under mild mechanical conditions.

Dry/neat grinding, are simple and commonly used processes for mechanochemical synthesis within solid blends ${ }^{14}$. These techniques have emerged as a useful, alternative technique to the traditional solvent-intensive methods for pharmaceutical cocrystal synthesis and production. However, there has been a number of reports presenting incomplete cocrystallisation using dry grinding ${ }^{15}$. Liquid-assisted grinding has, therefore, gained considerable favour because of the possibility of providing dramatically improved productivity via the addition of only small amounts of liquid to a typical grinding process ${ }^{16-24}$. It is, however, occasionally criticised for the unintentional production of cocrystal solvates ${ }^{25}$. In addition, the role of the added liquid differs from case to case, resulting in difficulties in clarifying reaction mechanisms ${ }^{16,20,26}$. More recently, another advanced solvent-free continuous manufacturing method, hot-melt extrusion (HME), has also emerged as an, easy to scale, alternative for mechanochemical cocrystal synthesis ${ }^{27-31}$. Interestingly, current literature has primarily focused on the preparation and characterization of novel cocrystals typically containing only the drug and coformer, leaving the subsequent formulation less explored ${ }^{32}$. IN this regard, Etter et al (1993) reported cocrystallisation in the presence of a third component by solid-state grinding 33 . The resulting product contained cocrystals of the complementary reagent pair, as well as the additional inert component that remained unchanged following cocrystal manufacture. Furthermore, cocrystals formed in the presence of the inert component had the same crystal structure as cocrystals grown from solution. More recently, driven by polymer-induced heteronucleation studies ${ }^{34}$, it has also been shown that the involvement of macromolecules in the cocrystal pool may also catalyse the reaction during mechanochemical preparation of cocrystals ${ }^{35}$. The cocrystals manufactured using polymer-assisted grinding methods were shown to negate the risk of generating undesirable solvates, while providing excellent control over the particle size of the resultant cocrsytals. Moreover, other important work has investigated cocrystal manufacture in the presence of an inert excipient ${ }^{36}$. The effects of coformers on phase 
transformation and release profiles of carbamazepine (CBZ) cocrystals in

106 hydroxypropyl methylcellulose (HPMC) matrix tablets were examined. It was

107 shown that HPMC partially inhibited the crystallisation of CBZ during dissolution.

108 If mechanochemical synthesis is to deliver its promise of being a clean 109 manufacturing technology, it must be shown to be capable of operating in an 110 environment devoid of solvent and be scalable. Furthermore, it is well 111 recognised that cocrystal synthesis is only one step in the development of 112 suitable oral dose formulations with other components (e.g., lubricant, glidant, 113 diluent) and unit operations (milling, sieving, blending, compression, filling) 114 being required before a successful drug product is produced. In light of the 115 above, we propose, for the first time, the use of hot melt extrusion, a solventless, 116 continuous and easily scalable technique, for the mechanochemical synthesis of 117 pharmaceutical cocrystals in the presence of a meltable binder. In this approach, 118 we examine chemically inert excipients that are amenable to hot melt extrusion, 119 forming a suspension of cocrystal particulates embedded in a pharmaceutical 120 matrix. We aim to understand if inert meltable carriers can be used to facilitate 121 the production of a solid extrudate while also acting as a catalyst for 122 cocrystallisation during melt-extrusion processing.

124 Selection of Formulation Components

125 Cocrystal Reagents

126 Ibuprofen (Ibu, Figure 1a) is a BCS Class II drug ${ }^{37}$ and has dissolution limited 127 absorption, particularly in acidic environment. Ibu has been widely used as a 128 cocrystal reagent, principally because it is inexpensive and contains a carboxylic 129 acid functional group that makes it an excellent donor to form intermolecular 130 hydrogen bonds with cocrystal reagents that possess a lone pair of electrons ${ }^{38}$.

131 Isonicotinamide (IsoNA, Figure 1a), although not classified as a GRAS 132 substance, has been shown to be an effective coformer with many literature 133 examples of carboxylic acid-IsoNA cocrystals ${ }^{39-41}$.

\section{Matrix Excipients}

136 Recently it has been demonstrated that a small molecular weight sugar alcohol, 137 mannitol, could be used as a matrix platform capable of significantly increasing 

the dissolution rate of poorly water soluble drugs ${ }^{42}$. In the work reported by

139 Thommes et al., (2011) HME was used to manufacture a suspension of 140 crystalline drug in a molten excipient to produce a uniform distribution of fine 141 particles. Rapid crystallization of mannitol 'fixed' the suspended drug particles 142 producing a solid homogeneous extrudate. In the work described in this article, 143 we adapt the concept of extruded crystalline suspensions and apply the 144 aforementioned preliminary criteria into matrix carrier selection. However, due 145 to thermal stability considerations, mannitol, which melts at $160^{\circ} \mathrm{C}$, was not used. 146 Consequently, xylitol which melts at a significantly lower temperature was 147 employed.

148 Despite the advantages sugar alcohols offer with respect to low melt viscosity and rapid solidification, extrudates may be difficult to shape post extrusion owing to the rigidity of their crystalline structure. Thus, the use of thermoplastic polymers was also examined. Eudragit ${ }^{\circledR}$ E PO and Soluplus ${ }^{\circledR}$ were chosen for such purposes owing to their relatively low $\mathrm{T}_{\mathrm{g}}$ and hence wider processing window. If cocrystals can be successfully manufactured and precipitated from an amorphous polymeric carrier, an amorphous suspension also referred to as a glass suspension could be formulated.

\section{Materials \& Methodology}

Materials

159 Ibuprofen, isonicotinamide, and xylitol were purchased from Sigma-Aldrich (St. 160 Louis, MO, USA). Eudragit ${ }^{\circledR}$ E PO was obtained from Evonik (Essen, Germany). 161 Soluplus ${ }^{\circledR}$ was kindly supplied by BASF Corporation (Ludwigshafen, Germany). 162 All other chemical reagents used were of analytical grade.

\section{Differential Scanning Calorimetry (DSC)}

165 Cocrystallisation feasibility studies and extrudate analyses were conducted on a 166 DSC 4000 (heat flux single furnace), and a DSC 8000 (power compensation dual 167 furnace), respectively (Perkin-Elmer, Windsor, Berkshire, UK). Both instruments 168 were calibrated at the respective ramp rates with indium and zinc for both 169 melting point and heat of fusion. Either dry nitrogen or helium was purged at a 170 flow rate of $40 \mathrm{~mL} / \mathrm{min}$ through the sample and reference cells to maintain an 
171 inert atmosphere. 3-5mg of sample was accurately weighed into an aluminium 172 pan and crimped using an aluminium pan lid. The crimped pan set was then 173 subjected to a thermal ramp at $20^{\circ} \mathrm{C} / \mathrm{min}$ in DSC 4000 , and $200^{\circ} \mathrm{C} / \mathrm{min}$ in DSC 1748000 , respectively, from $-60^{\circ} \mathrm{C}$ to $200^{\circ} \mathrm{C}$. The polymeric candidates were 175 subjected to modulated DSC (TA Q100, TA Instruments) at $2^{\circ} \mathrm{C} / \mathrm{min}$, with an 176 amplitude and frequency of $\pm 0.6^{\circ} \mathrm{C}$ every 40 s to enable the determination of the 177 glass transition temperature $\left(\mathrm{T}_{\mathrm{g}}\right)$.

\section{Thermogravimetric Analysis (TGA)}

180 The decomposition temperature for each individual substance was determined 181 using a Thermal Advantage Model Q500 TGA (TA instruments, Leatherhead, UK). 182 Ramp tests were performed on powdered samples (5-10 mg) heated at $18310^{\circ} \mathrm{C} / \mathrm{min}$ over a range from $0^{\circ} \mathrm{C}$ to $400^{\circ} \mathrm{C}$. Dry nitrogen (flow rate sample: 60 $184 \mathrm{~mL} / \mathrm{min}$, flow rate balance: $40 \mathrm{~mL} / \mathrm{min}$ ) was purged through the sample 185 chamber during all experiments to maintain an inert environment and hence 186 prevent oxidation. The temperature at which a 5\% weight loss occurred was 187 recorded for each sample and considered as the onset of material decomposition.

\section{Preparation of the Reference Cocrystal Standard}

1900.01 moles of equimolar ibuprofen-isonicotinamide mixture was dissolved in $19150 \mathrm{~mL}$ methanol and stirred at room temperature until complete dissolution was 192 achieved. The resulting clear solution was left in a fume hood covered with a 193 funnel to allow slow evaporation of the solvent for 48 hours. The precipitate was 194 collected and subsequently stored in an oven at $45^{\circ} \mathrm{C}$ for a further 24 hours to 195 remove any residual solvent. The resulting material was gently pulverized using 196 a mortar and pestle, sized through a $220 \mu \mathrm{m}$ sieve and stored in a vacuum 197 desiccator before being subjected to further analysis.

\section{Cocrystallisation via Ball-Milling}

200 Equimolar ibuprofen-isonicotinamide mixtures with or devoid of excipient were 201 ground using a ball mill (MM200, Retsch, Reinische, Haan, Germany) at a 202 frequency of $20 \mathrm{~s}^{-1}$ frequency for pre-determined periods of time (i.e. 2, 5, 8, 15, 
30,45 and 60 minutes). The resulting pulverized mixtures were sized through a

$204220 \mu \mathrm{m}$ sieve before being subjected to further characterisation.

\section{Cocrystallisation via Hot-Melt Extrusion}

207 Physically mixed blends of each formulation were manually fed into a co-rotating twin-screw HAAKE Mini-lab Extruder (HAAKE Minilab, Thermo Electron Corporation, Stone, Staffordshire, UK). The process temperature was determined according to the melting temperature(s) of the crystalline compound(s) in the

211 physical blend with screw speed set at 10rpm. For the formulations containing xylitol, the HME parameters were slightly modified to prevent early-stage phase separation and late-stage die blockage. In particular, the processing was divided

214 into two stages: the 'feeding stage' where temperature was set at the melting 215 point of the polyol and screw speed set at 10rpm; and the 'flushing stage' where

216 temperature was set $7^{\circ} \mathrm{C}$ lower than the polyol melting temperature and screw speed set at 50rpm (Table 1). All collected products were pulverized by mortar and pestle and subsequently stored in a desiccator over silica gel at room temperature prior to further analyses.

\section{Powder X-ray Diffraction (PXRD)}

222 Samples were analysed at room temperature using a MiniFlex II Desktop Powder 223 X-ray Diffractometer (Rigaku Corporation, Kent, England) equipped with Cu K $\beta$ 224 radiation, at a voltage of $30 \mathrm{kV}$ and a current of $15 \mathrm{~mA}$. The powdered samples 225 were gently consolidated on a glass top-loading sample holder with $0.2 \mathrm{~mm}$ 226 depression. All samples were scanned within the angular range of $1.5-40^{\circ} 2 \Theta$ in continuous mode with a sampling width of $0.03^{\circ}$ and a scan speed of $2.0^{\circ} / \mathrm{min}$.

\section{Quantification of Cocrystal Yielding}

230 The peak area of the cocrystal characteristic peak at $3.3^{\circ} 2 \Theta$ for each sample was 231 used to determine the cocrystal yield ${ }^{43}$. A series of physical mixtures containing 232 the reference cocrystal and xylitol at 10 different cocrystal loadings, 10, 20, 30, $23340,50,60,70,80,90 \& 100 \% \mathrm{w} / \mathrm{w}$, respectively, were prepared through gentle 234 mixing. The blended samples were placed into a $0.2 \mathrm{~mm}$-deep squared 235 indentation on the glass sample holder for PXRD analysis. The integration region 
236 was set between [2.400 4.050 $2 \theta$ ] with manual background subtraction using

237 the IntegralAnalysis Version 6.0 (Rigaku Corporation, Kent, England). A 238 calibration curve, $y=284.877 x-668.959\left(R^{2}=0.998\right)$ was constructed using linear 239 regression of the average peak area against theoretical cocrystal concentration 240 in the blends. The calibration curve was validated for linearity, accuracy, 241 precision, LoD and LoQ according to the methods recommended in the ICH 242 guidelines ${ }^{44}$ (data included as supporting documents).

\section{FT Infrared Spectroscopy (FTIR)}

245 FT-IR spectroscopy was used to investigate molecular interactions and to 246 identify the structure of Ibu/IsoNA cocrystal. Experiments were performed using 247 a Fourier transform infrared spectrophotometer model 4100 (FT/IR-4100) 248 (Jasco, Easton, MD), incorporated with the Version 2 Jasco Spectra Manager 249 Software. A scanning range of $4000-400 \mathrm{~cm}^{-1}$ with $4.0 \mathrm{~cm}^{-1}$ resolution and 16 250 scans per spectrum was used for all samples. Prior to FTIR spectroscopic 251 analysis, samples were gently ground with dry potassium bromide (KBr) powder 252 using an agate mortar and pestle and compressed at approximately 7.5Pa for 60s 253 to prepare a $\mathrm{KBr}$ disk. The concentration of the samples in a $\mathrm{KBr}$ disk was 254 maintained at $0.67 \%$ (2mg sample plus $298 \mathrm{mg} \mathrm{KBr}$ ) for all analyses.

\section{Raman Microscopy \& Mapping}

257 Raman spectroscopic analyses were conducted using a RamanMicro 300 Raman 258 microscope (Perkin Elmer, Windsor, Berkshire, UK) coupled with an Avalon 259 Raman station R3 Model AVRS003A spectrometer (Avalon Instruments, Belfast, 260 UK). A magnification of $20 \mathrm{x}$ with a total exposure time of 20 s (4s acquisition $\times 5$ ) 261 was used for all samples. Data was collected from $200-3200 \mathrm{~cm}^{-1}$ and analysed 262 using Spectrum v6.3.4 software with an automatic baseline correction. Cross 263 sections of rod shaped extrudates were mapped with a $50 \mu \mathrm{m}$ spacing between 264 each sampling point. Approximately 4000 points were collected across the 265 exposed mapping area for the cross section of one pellet. The laser power was 266 set at $80 \%$ throughout the mapping process to avoid sample saturation. 267 Spectrum IMAGE R1.6.4.0394 software was used to conduct mapping analysis 268 for each examined sample. The characteristic peak associated with the 
$269 \mathrm{Ibu} /$ IsoNA cocrystal at $1020 \mathrm{~cm}^{-1}$ was used in the single wavenumber mode. The 270 maps were shown with an ordinate axis range of [1000-7500 INT], whilst the

271 horizontal $X$ axis range and the vertical $Y$ axis range were both 0-2350 $\mu \mathrm{m}$. A

272 rainbow cubic look-up table was utilized to illustrate the intensity of the chosen 273 cocrystal peak.

\section{Polarized Light Microscope (PLM)}

276 A polarized light microscope (Olympus BX50F4, Microscope Service and Sales, 277 Surrey, UK) was used to study the morphology of the melt-extruded cocrystals in 278 comparison to that of the unprocessed ibuprofen. Polarized light micrographs of 279 each sample were captured at room temperature using a Pixelink Megapixel 280 Firewire Camera and Pixelink software (Scorpion Vision Ltd., Lymington, UK). 281 The milled sample within the size range 180 212 $\mu \mathrm{m}$ was dispersed in a drop of 282 mineral oil on a glass slide. All measurements were performed at a magnification 283 of $200 x$ with the polariser and analyser positioned perpendicularly.

\section{In-vitro Dissolution Study}

286 In-vitro drug dissolution tests were conducted to evaluate solubility enhancement and dissolution behaviour of the melt extruded Ibu/IsoNA cocrystals in comparison with that of the unprocessed ibuprofen. Release studies were performed using a Caleva dissolution tester 10ST (GB Caleva Ltd., Dorset, UK) according to the BP 2011 apparatus II, paddle method. The powdered

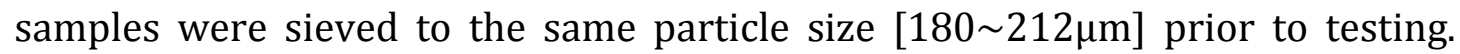
Each formulation, containing equivalent to $120 \mathrm{mg} \mathrm{Ibu}$, was tested in $600 \mathrm{~mL}$ deionized water at $37 \pm 0.5^{\circ} \mathrm{C}$ using a paddle rotation speed of $75 \mathrm{rpm} .3 \mathrm{~mL}$

294 aliquots were withdrawn from each dissolution vessel at regular time intervals 295 and filtered through a $0.45 \mu \mathrm{m}$ Millipore filter unit (MILLEX ${ }^{\circledR}$-GS, Millipore, 296 Carrigtwohill Co, Cork, Ireland) before being subjected to a validated HPLC 297 analysis. Immediately after sample withdrawal, $3 \mathrm{~mL}$ of blank dissolution media 298 was added into each vessel to maintain the overall media volume. 
301 The concentration of ibuprofen in each sampled aliquot was determined using 302 HPLC analysis. The HPLC system consisted of a Waters binary HPLC pump 1525, 303 a Plus Auto sampler 717, an In-line Degasser AF and a Dual $\lambda$ Absorbance 304 Detector 2487 (Waters, Massachusetts, USA). Sampled aliquots were analysed at $305220 \mathrm{~nm}$ using a Jupiter C18 300 column $(5 \mu \mathrm{m})$ with a length of $250 \mathrm{~mm}$ and a 306 diameter of $4.60 \mathrm{~mm}$ (Phenomenex, Macclesfield, UK). The mobile phase 307 consisted of $85 \%$ methanol and $15 \%$ deionized water containing $0.2 \%$ TFA. The 308 flow rate was set at $1 \mathrm{~mL} / \mathrm{min}$ and the column chamber was maintained at $40^{\circ} \mathrm{C}$ 309 for the entire analytical procedure. The average retention times under these 310 conditions were 4.56 minutes for Ibu, 3.40 minutes for IsoNA, while other 311 components were tested to show no interference. When Soluplus ${ }^{\circledR}$ was involved, 312 the mobile phase was changed to a 70:30 ratio between the organic and 313 inorganic solutions with a retention time of 8.76 minutes for ibuprofen to avoid 314 interference. Peak areas were calculated using Breeze 3.30 software. Standard 315 solutions were prepared in triplicate using methanol and deionized water at 1:1 316 volume ratio for the generation of a linear calibration curve $\left(R^{2}>0.999\right)$. The 317 calculated concentrations of ibuprofen dissolved during the dissolution test were 318 then plotted as a function of time.

\section{Storage Stability}

321 The extruded suspensions were stored either in a desiccator over silica gel for 12 322 months under ambient conditions or in a desiccator over saturated sodium 323 chloride solution at $20^{\circ} \mathrm{C}$ and $70 \% \mathrm{RH}$ for 12 months. The aged suspensions were 324 examined by PXRD and the cocrystal content in each formulation was calculated 325 using the aforementioned yielding quantification.

\section{Statistical Analysis}

328 Statistical analyses were conducted using a one-way analysis of variance 329 (GraphPad Prism 6.0). Individual differences between treatment groups were 330 identified using Tukey's post-hoc test with $P<0.05$ denoting statistical 331 significance. 


\section{Formation of Ibu/IsoNA Cocrystal}

335 In this work we considered that Ibu and IsoNA would form a cocrystal at an 336 equimolar stoichiometric ratio ${ }^{45-48}$. This would be facilitated by the interaction 337 of the carboxylic acid functional group of Ibu, the IsoNA amide and the $\mathrm{N}$ on the 338 pyridine of IsoNA ${ }^{26}$. With respect to IBu and IsoNA, the carboxylic acid group, would be highly pertinent in successful formation of cocrystal with carboxylic acid-aromatic nitrogen and carboxylic acid-amide synthons being the major interactions present (Figure 1b).

Prior to addition of pharmaceutical excipients and evaluation of extrusion as a continuous process for mechanochemical synthesis of cocrystals, we employed ball milling to determine the feasibility of forming a cocrystal product from an Ibu/IsoNA blend at a 1:1 molar ratio. Conventional DSC analysis at a heating rate of $20^{\circ} \mathrm{C} / \mathrm{min}$ was used to confirm the formation of the Ibu/IsoNA cocrystal. It has been previously reported in many articles that the melting temperature $\left(\mathrm{T}_{\mathrm{m}}\right)$ of cocrystal is often between that of the drug and the coformer, or lower than both individual $\mathrm{T}_{\mathrm{m}}$ values. The DSC thermograms for Ibu, IsoNA, and their equimolar physical mixtures are shown in Figure 2. Ibu and IsoNA exhibited characteristic melting points, when heated using a ramp rate of $20^{\circ} \mathrm{C} / \mathrm{min}$, at approximately $80.58 \pm 0.32^{\circ} \mathrm{C}$ and $161.56 \pm 0.59^{\circ} \mathrm{C}$, respectively. Physically mixed and ball-milled samples exhibited DSC traces devoid of an endothermic peak characteristic of IsoNA melting and importantly, a new endothermic event, considered to be the heat of fusion for a cocrystal was observed at approximately $123^{\circ} \mathrm{C}$. The enthalpy associated with this peak increased in value as a result of increasing mechanical energy (physically mixed sample $(50.1 \pm 0.77 \mathrm{~J} / \mathrm{g}) ; 2$ minutes milling $(148.6 \pm 0.83$ $\mathrm{J} / \mathrm{g}) ; 5$ minutes milling $(155.6 \pm 1.02 \mathrm{~J} / \mathrm{g})$ ). Moreover, ball milled samples exhibited an Ibu melting peak that was significantly depressed, decreasing from $80.58 \pm 0.32^{\circ} \mathrm{C}$ to $73.97 \pm 0.56^{\circ} \mathrm{C}$ and $72.03 \pm 0.04^{\circ} \mathrm{C}$ for 2 minutes and 5 minutes milling, respectively. The enthalpy of these two transitions was also significantly lowered. Moreover, there was no evidence of a melting endotherm for crystalline IsoNA in the DSC thermograms where Ibu was present. From the data, it would appear that once Ibu melted, IsoNA dissolved in the molten drug. 
366 value of enthalpy associated with the cocrystal peak from $148.6 \pm 0.83 \mathrm{~J} / \mathrm{g}$ to

$36762.46 \pm 0.64 \mathrm{~J} / \mathrm{g}$ after 2 minutes milling. In this case it is important to note that the 368 maximum enthalpy expected for the formulations incorporating $10 \% \mathrm{w} / \mathrm{w}$ 369 xylitol would be approximately $133 \mathrm{~J} / \mathrm{g}(0.9 \mathrm{x} 148.6)$. The enthalpy observed at 2 370 minutes milling in the presence of 10\% Xylitol was approximately $48 \%$ of what 371 was expected. The enthalpy, nonetheless, increased significantly with increasing 372 duration of milling, reaching $124.69 \pm 1.13 \mathrm{~J} / \mathrm{g}$ (96\% of value observed for neat 373 cocrystallisation) after 45 minutes of consecutive milling. A further 15 minutes 374 milling processing, however, decreased the measured cocrystal $\Delta \mathrm{H}$ to $375107.25 \pm 0.98 \mathrm{~J} / \mathrm{g}$. In all cases, there was a clear melting endotherm present for 376 Xylitol suggesting that, unlike IsoNA, it remained crystalline following Ibu 377 melting.

\section{Hot Melt Extrusion}

380 Hot melt extrusion is a non-ambient process that forces materials through a 381 heated barrel. Processing parameters and formulation variables have a 382 significant impact upon the properties of extruded product. The process 383 parameters and associated observations made during HME are listed in Table 1. 384 The incorporation of polymeric matrix carriers (formulations 2 and 3 ) 385 significantly reduced the torque values, when compared with formulation 1 (Net 386 cocrystal reagents). The addition of $10 \% \mathrm{w} / \mathrm{w}$ xylitol resulted in an increase of 387 torque from $40-44 \mathrm{Ncm}$ up to $109-122 \mathrm{Ncm}$ and a reduction in the residence time 388 from 233s to $90 \mathrm{~s}$. A further increase in the xylitol concentration to $30 \% \mathrm{w} / \mathrm{w}$ 389 decreased the torque to $43-59 \mathrm{Ncm}$, similar to the torque values recorded for 390 formulation 1. A further increase in the concentration of xylitol to $50 \% \mathrm{w} / \mathrm{w}$ 391 reduced the torque to $19-22 \mathrm{Ncm}$. Interestingly, decreasing torque values 392 progressing through formulations 4, 5 and 6 resulted in residence times that 393 were increased. The addition of xylitol (30\% and 50\%) increased residence time 394 to 272 s and 329 s, respectively.

\section{Thermal Analysis}

397 When using conventional DSC at a heating rate of $20^{\circ} \mathrm{C} / \mathrm{min}$ during preliminary 398 study, physically mixed Ibu and IsoNA blends showed only endothermic events 

typical of the melting of Ibu and the Ibu/IsoNA cocrystal (Figure 2). By using hyper DSC $\left(200^{\circ} \mathrm{C} / \mathrm{min}\right)$, however, it was possible to observe an endothermic peak characteristic of the melting of the residual IsoNA content. The thermograms provided by hyper DSC measurements for pure Ibu, IsoNA, xylitol and their extruded mixtures are presented in Figure 4. Ibu and IsoNA exhibited characteristic melting points (peak) at $84.41 \pm 0.57^{\circ} \mathrm{C}$ and $163.26 \pm 0.99^{\circ} \mathrm{C}$, respectively, when heated using a ramp rate of $200^{\circ} \mathrm{C} / \mathrm{min}$. The DSC trace obtained for the reference cocrystal, precipitated from solution, showed one single endothermic event, typical of a melting transition ranging from $120.78 \pm 0.03^{\circ} \mathrm{C}$ to $134.59 \pm 0.43^{\circ} \mathrm{C}$, with the peak maximum at $127.29 \pm 0.55^{\circ} \mathrm{C}$.

At such relatively fast heating rate, xylitol melted at $104.17 \pm 0.29^{\circ} \mathrm{C}$ and displayed a broad melting peak ranging from $92.98 \pm 0.13^{\circ} \mathrm{C}$ to $112.30 \pm 0.17^{\circ} \mathrm{C}$. The similarity of melting temperatures for xylitol and the cocrystal presented difficulty in using DSC to determine presence of cocrystal. This was made more difficult by the fact that the melting transition associated with the cocrystal showed considerable depression if measured in the presence of xylitol (Figure 4). This was particularly relevant in formulations containing high concentrations of xylitol. Moreover, evidence of unreacted Ibu and IsoNA could be observed in thermograms associated with suspensions of $30 \%$ and $50 \%$ xylitol. However, $\mathrm{T}_{\mathrm{m}}$ of the residue of both parent components were also noticeably depressed (both onset and end point).

Amorphous carriers Eudragit ${ }^{\circledR}$ E PO and Soluplus ${ }^{\circledR}$ showed a $\mathrm{T}_{\mathrm{g}}$ at $43.94 \pm 0.13^{\circ} \mathrm{C}$ and $66.52 \pm 0.20^{\circ} \mathrm{C}$, respectively (Table 2). All carrier excipients studied were thermally stable whereas ibuprofen and the reference cocrystal showed onset of decomposition at $197.44 \pm 3.67^{\circ} \mathrm{C}$ and $164.07 \pm 6.77^{\circ} \mathrm{C}$, respectively.

\section{PXRD}

PXRD patterns are depicted in Figure 5. Ibu showed distinct peaks at $6.3^{\circ}$, $16.7^{\circ}, 20.2^{\circ}$ and $22.4^{\circ} 2 \Theta$, ISoNA at $17.9^{\circ}, 21.0^{\circ}$ and $23.4^{\circ} 2 \Theta$, and Xylitol at $20.0^{\circ}$, $22.5^{\circ}, 22.7^{\circ}$ and $38.4^{\circ} 2 \Theta$, respectively. For the physically mixed systems the PXRD data (iv) correlated well with DSC data in that there was little evidence of cocrystal formation. For the extruded formulations containing Xylitol, the 
cocrystal product $(\mathrm{V}-\mathrm{X})$ showed distinct peaks that were distinguishable from

433 simple overlap of cocrystal reagents and xylitol. New peaks were evident at $3.3^{\circ}$ 434 and $17.1^{\circ} 2 \Theta$. The intensity of the peaks attributable to cocrystal product varied 435 as a function of excipient type and concentration. It is evident from cocrystal 436 yield that the conversion from the parent reagents to the cocrystal was $43728.06 \pm 1.65 \%, 33.46 \pm 0.55 \%, 28.60 \pm 0.61 \%$ and, $28.25 \pm 0.65 \%$ for formulations 1 , 4384,5 and, 6, respectively. Those formulations extruded with polymeric excipient, 439 on the other hand, showed no evidence of cocrystal formation. Interestingly, 440 these diffractograms (vi and vii) showed no characteristic peaks attributable to 441 Ibu either. This would suggest that Ibu had been rendered amorphous following extrusion.

\section{FTIR Spectroscopic Analysis}

445 The FTIR spectra, in the $3600-2600 \mathrm{~cm}^{-1}$ and $2000-1200 \mathrm{~cm}^{-1}$ wavenumber 446 intervals, for Ibu, IsoNA, xylitol, the cocrystal reference, and the extruded suspensions containing 10\%, 30\% and, 50\% xylitol, respectively, are represented in Figure 6. The assignment of IR vibrational bands in Ibu, IsoNA and the equimolar reference cocrystal obtained from solution method is listed in Table 3. The FTIR spectrum for Ibu showed a number of weak peaks in the wavenumber region [3100-2900 $\mathrm{cm}^{-1}$ ] reflecting complex modes of vibrations associated to $\mathrm{C}-\mathrm{H}, \mathrm{C}-\mathrm{H}_{2}$ and $\mathrm{C}-\mathrm{H}_{3}$ groups $^{49}$; and a very broad peak covering almost the whole wavenumber range from $3400 \sim 2800 \mathrm{~cm}^{-1}$ associated to $\mathrm{O}-\mathrm{H}$ stretching vibrations within a carboxylic acid dimeric structure of Ibu. A sharp and intense $\mathrm{C}=0$ stretching band at $1721.16 \mathrm{~cm}^{-1}$ was also observed for Ibu due to the presence of a mono carboxylic acid group 50 . IsoNA, on the other hand, showed characteristic IR bands at: (i) $3369.03 \mathrm{~cm}^{-1}$ and $3185.83 \mathrm{~cm}^{-1}$,

458 representing asymmetric and symmetric $v_{\mathrm{N}-\mathrm{H}}$ stretching vibrations, respectively, 459 for the H-bonded primary amide groups among closely packed IsoNA 460 molecules 51,52 ; (ii) $1668.12 \mathrm{~cm}^{-1}$, denoting $v_{\mathrm{C}=0}$ stretching of the amide carbonyl 461 group 53 ; (iii) $1622.80 \mathrm{~cm}^{-1}$ and $1594.84 \mathrm{~cm}^{-1}$ for the $\delta_{\mathrm{N}-\mathrm{H}}$ bending vibrations of 462 the primary amide ${ }^{54}$; and (iv) $1551.45 \mathrm{~cm}^{-1}$ signifying $v_{\mathrm{C}=\mathrm{N}}$ ring stretching in the 463 heterocyclic pyridine ring structure ${ }^{54,55}$. 
464 As shown in Figure 1, the the cocrystal structure involves a number of groups 465 with characteristic IR vibrational bands. They are the IsoNA amide N-H 466 (stretching and bending), the ISoNA amide $\mathrm{C}=\mathrm{O}$ (stretching), and the pyridine $\mathrm{N}$ 467 of isoNA, as well as the carboxylic acid group of IBU.

468 The amide carbonyl and the pyridine $\mathrm{N}$ are two competing H-bond acceptor 469 sites in IsoNA structure. The pyridine $\mathrm{N}$, however, is generally considered a 470 better acceptor 56 , and hence more prone to attract the amide $\mathrm{H}$-atom, forming a $471 \mathrm{~N}-\mathrm{H} \bullet \bullet \mathrm{N}$ bond in bulk IsoNA. In forming an amide homodimer synthon during 472 cocrystallisation, the amide N-H asymmetric and symmetric stretching (3369.03 $473 \mathrm{~cm}^{-1}$ and $3185.83 \mathrm{~cm}^{-1}$ ) were shifted to $3434.60 \mathrm{~cm}^{-1}$ and $3173.29 \mathrm{~cm}^{-1}$, 474 respectively, whilst the amide carbonyl stretching vibration band $\left(1668.12 \mathrm{~cm}^{-1}\right)$ 475 was shifted to $1629.55 \mathrm{~cm}^{-1}$. The $\mathrm{N}-\mathrm{H}$ blue shift to $3434.60 \mathrm{~cm}^{-1}$ indicated 476 dissociation of the existing $\mathrm{N}-\mathrm{H} \bullet \bullet \mathrm{N}$ bond, generating free amide $\mathrm{N}-\mathrm{H}$. The $\mathrm{N}-\mathrm{H}$ 477 red shift to $3173.29 \mathrm{~cm}^{-1}$, together with the carbonyl red shift to $1629.55 \mathrm{~cm}^{-1}$, 478 were both attributed to the formation of $\mathrm{N}-\mathrm{H} \bullet \bullet \bullet \mathrm{O}$ bonds between the amide $\mathrm{N}-\mathrm{H}$ 479 and amide carbonyl groups in the IsoNA homodimer.

480 The lone pair of electrons on the pyridine $\mathrm{N}$, after dissociation of the original $481 \mathrm{~N}-\mathrm{H} \bullet \bullet \mathrm{N}$ bond in bulk IsoNA, provides a strong proton acceptor site. The absence 482 of the associated Ibu $0-\mathrm{H}$ stretch (broad peak in the region $3400 \sim 2800 \mathrm{~cm}^{-1}$ ) 483 provides support for dimeric dissociation in bulk Ibu. The occurrence of an 484 additional peak at $3317.93 \mathrm{~cm}^{-1}$ was attributed to the formation of a 485 supramolecular heteromeric synthon through $\mathrm{O}-\mathrm{H} \bullet \bullet \mathrm{N}$ hydrogen bonding 486 between the pyridine $\mathrm{N}$ and the Ibu $0-\mathrm{H}^{38}$. Moreover, in forming the carboxylic 487 acid-pyridine H-bond, the carboxylic acid carbonyl $\left(1721.16 \mathrm{~cm}^{-1}\right)$ was red 488 shifted to $1702.84 \mathrm{~cm}^{-1}$, while a C-H stretching $\left(796.457 \mathrm{~cm}^{-1}\right)$ from the pyridine 489 ring was also red shifted to $779.101 \mathrm{~cm}^{-1}$, indicating formation of a $\mathrm{C}-\mathrm{H} \bullet \bullet 0$ 490 hydrogen bond within the heteromeric synthon ${ }^{39,57}$. It was also apparent that the 491 addition of xylitol as a matrix carrier did not alter interactions between two 492 parent cocrystal reagents. The IR spectra of the cocrystal/xylitol suspensions 493 were typical of the spectrum of cocrystal with xylitol superimposed (Figure 6). 494 
As shown in Figure 7, the unprocessed Ibu, IsoNA, and their equimolar cocrystal showed three distinctive peaks within Raman shift region $1050.0 \sim 975.0 \mathrm{~cm}^{-1}$. 498 Ibu exhibited a peak at $1006.13 \mathrm{~cm}^{-1}$ characteristic of aromatic ring C-C stretching. IsoNA presented a very intense and well-defined peak at $993.97 \mathrm{~cm}^{-1}$ attributed to the pyridine ring structure. In the equimolar cocrystal, the pyridine peak was broadened and the wavenumber was shifted to $1020.50 \mathrm{~cm}^{-1}$. There were two shoulders characteristic of the vibration of the aromatic ring of Ibu, as well as the residual pyridine ring structure from the remaining free IsoNA. The matrix carrier xylitol, on the other hand, did not show any distinctive peak within this Raman shift region. It is, therefore, clear that the peak at $1020.50 \mathrm{~cm}^{-1}$, characteristic of the cocrystal, is free of interference from any other component within the formulation. By plotting the intensity of this specific peak across the the sampled cross-sectional area of the extrudate we can determine the distribution of the cocrystal.

510 Figure 8 (a) provides a Raman map of the extrudate produced using only 511 cocrystal reagents devoid of any excipient. The peak intensity at $1020 \mathrm{~cm}^{-1}$ has 512 been used to generate the Raman map using a rainbow cubic look-up table. The 513 lookup table was generated within an ordinate range of [1000-7500 INT]. It is 514 apparent that there is a difference in the intensity of the cocrystal peak across 515 the cross-sectional area with a highly intense region focused to the outer edges 516 of the extrudate. The spectra of a number of labelled points, representing a range 517 of intensities across the map are provided for comparative purposes.

518 The mapping results shown in Figure 9 depict the intensity values of the $5191020.50 \mathrm{~cm}^{-1}$ peak in extrudates containing different concentrations of xylitol 520 and following storage. Interestingly, the intensity of the cocrystal peak varied 521 considerably as a function of xylitol concentration and storage. When extruded 522 with 10\% xylitol (Figure 9(1)), there was an obvious and significant increase in 523 the intensity of cocrystal (relative to neat extrusion) across the cross-sectional 524 area of the extrudate. This result correlated well with results obtained from XRD 525 where the formulations containing 10\% xylitol had intense diffraction bands at $5263.3^{\circ}$ and $17.1^{\circ}$. With increasing xylitol concentration in the formulation $(30 \%$ 527 and $50 \%$, respectively), the overall intensity for the characteristic cocrystal peak 
528 at $1020.50 \mathrm{~cm}^{-1}$ decreased throughout the entire cross-section (Figure 9, (2) and

529 (3)). This result was again mirrored in the XRD where the cocrystal yield 530 decreased from $33.46 \pm 0.55 \%$ to $28.25 \pm 0.65 \%$, as the concentration of xylitol 531 increased from $10 \%$ to $50 \%$. Importantly, after 12-month storage under 532 controlled conditions $\left(20^{\circ} \mathrm{C}\right.$ with $\left.70 \% \mathrm{RH}\right)$, an extensive increase in the intensity 533 values for the distinctive cocrystal peak at $1020 \mathrm{~cm}^{-1}$ was observed for all three 534 formulations containing xylitol (Figure 9, (4), (5) \& (6)), suggesting significant 535 growth of the cocrystal content upon aging.

\section{Cocrystal Morphology Study}

538 The crystal habit of a drug is extremely important consideration in 539 pharmaceutical manufacturing. Typically a number of basic physicochemical 540 properties such as solubility, dissolution rate, powder flow, compressibility, and 541 mechanical strength depend on the crystal habit. Figure 10 cshows polarised 542 light micrographs of $\mathrm{Ibu}$, as received, cocrystal formed using traditional solvent 543 methods and melt extruded cocrytal particles. The PLM images clearly depict the 544 needle-like shape of both Ibu and the reference cocrystal standard. Such 545 anisotropic shape could be problematic during pharmaceutical manufacturing 54658,59 . Conversely, the melt-extruded cocrystal particles (Figure 10c), were 547 uniformally shape and much smaller in size.

\section{In-vitro Drug Dissolution}

550 Drug release profiles (Figure 11) and associated data confirmed that all extruded

551 formulations exhibited improved solubility and increased dissolution rate of Ibu 552 with the exception of the formulation containing Eudragit ${ }^{\circledR}$ E PO. The amorphous 553 dispersion with Soluplus ${ }^{\circledR}$ (formulation 2) had a similar relative dissolution rate 554 at $5 \mathrm{~min}(0.95 \pm 0.06)$ and $45 \mathrm{~min}(0.44 \pm 0.04)$ to the neat-extruded formulation 1 $555(0.94 \pm 0.04$ and $0.44 \pm 0.00$, respectively). Formulation 1 , however, had a 556 significantly higher dissolution rate $(0.20 \pm 0.01)$ and increased solubility 557 (DP180min of $36.30 \pm 1.33 \%$ ) at $180 \mathrm{~min}$ than formulation $2(0.16 \pm 0.02$, and $55829.08 \pm 3.13 \%$, respectively). The inclusion of xylitol significantly increased the 559 dissolution rate at drug percent released. In particular, the extruded suspension 560 containing $50 \% \mathrm{w} / \mathrm{w}$ xylitol (formulation 6) exhibited the highest DP and RDr 
561 values of all formulations $\left(\mathrm{DP}_{5 \mathrm{~min}}\right.$ at $6.41 \pm 0.07 \%, \mathrm{DP}_{45 \mathrm{~min}}$ at $37.41 \pm 0.81 \%$,

$562 \mathrm{DP}_{180 \mathrm{~min}}$ at $43.53 \pm 0.34 \%, \mathrm{RDr}_{5 \mathrm{~min}}$ of $1.28 \pm 0.01, \mathrm{RDr}_{45 \mathrm{~min}}$ of $0.83 \pm 0.02$ and,

$563 \mathrm{RDr}_{180 \mathrm{~min}}$ of $0.24 \pm 0.00$, respectively.

\section{Cocrystal Content Evaluation After Aging}

566 The stability of pharmaceutical materials that may undergo physical form change 567 during storage is fundamentally important. Stability must be examined in detail 568 in order to develop successful pharmaceutical products. The physical stability 569 relating to the relative quantity of cocrytal is shown in Figure 12. Samples were 570 stored at room temperature under both desiccated and humid conditions $\left(20^{\circ} \mathrm{C}\right.$ 571 over silica gel, and $20^{\circ} \mathrm{C}$ with $70 \% \mathrm{RH}$, respectively) to evaluate changes in the 572 cocrystal content. As previously presented, formulation 4 that contained 10\% $573 \mathrm{w} / \mathrm{w}$ xylitol showed a significantly higher cocrystal yield $(33.46 \pm 0.55 \%)$ than the 574 neat-extruded formulation $1(28.06 \pm 1.65 \%)$ immediately after HME processing. 575 Increasing the concentration of xylitol considerably decreased the yield for 576 formulations $5(28.60 \pm 0.61 \%)$ and $6(28.25 \pm 0.65 \%)$, respectively. A similar but 577 qualitative indication was also evident in the Raman maps. Interestingly, the 578 neat extruded formulation 1 showed an increase in cocrystal yield increase 579 following 12-month storage under dry (33.85 $\pm 2.69 \%)$ and humid (43.31 $\pm 1.25 \%)$ 580 conditions, respectively. Formulations containing xylitol, retained the same 581 quantity of cocrystal yield following storage under dry conditions, while showing 582 increase to various extents, $34.86 \pm 0.85 \%, 35.66 \pm 1.88 \%$ and $34.49 \pm 2.40$ for 583 formulations 4, 5 and 6, respectively, following storage under humid conditions.

\section{Discussion}

586 Pharmaceutical cocrystals offer a way to overcome the solubility issues 587 associated with BCS class II compounds while also retaining the thermodynamic 588 stability of the crystalline form of a drug60,61. Conventionally, the manufacture of 589 a pharmaceutical cocrystal product is divided into two major aspects: the 590 production/synthesis of the cocrystal itself and the subsequent formulation of 591 cocrystal into a pharmaceutical dosage form. Pharmaceutical cocrystals have 592 developed significantly in the past decade with increasing number of patents 593 issued worldwide. However, at present there is still limited marketed examples 
594 of pharmaceutical products involving cocrystals ${ }^{62,63}$. With the recent 595 implementation of techniques such as hot-melt extrusion ${ }^{28}$ and spray drying 64,65 , 596 as methods of manufacturing pharmaceutical cocrystals, it has become possible 597 to combine cocrystallisation and formulation to reduce the number of 598 manufacturing steps involved in drug product manufacture. Hot-melt extrusion 599 is particularly advantageous owing to its continuous processing capability; ease 600 of scaling and it may be used to manufacture cocrystals without the need for 601 organic solvents.

602 To achieve mechanochemical synthesis of cocrystals via HME comprehensive 603 investigations must be conducted in order to develop a thorough understanding 604 of the reaction selectivity between ingredients; the most appropriate reaction 605 conditions/parameter settings for a specific system; and most importantly, the 606 selection of a suitable matrix for the cocrystal reagents. To probe this further, we 607 have processed mixtures consisting of chosen cocrystal reagents and an 'inert' 608 carrier excipient via hot melt extrusion. The principal hypothesis being that $609 \mathrm{Ibu} /$ IsoNA cocrystals should be suspend in the final matrix. Consequently, the 610 HME processing temperature was set significantly lower than the $\mathrm{T}_{\mathrm{m}}$ onset of the 611 cocrystals (approximately $120^{\circ} \mathrm{C}$ ). The chosen carrier excipient was either a 612 pharmaceutical grade polymer (Eudragit ${ }^{\circledR}$ EPO and Soluplus ${ }^{\circledR}$ ) with relatively 613 low values of Tg hence lower processing temperature, or a commonly used food 614 and pharmaceutical additive, namely Xylitol, that was molten at the chosen 615 extrusion temperature.

616 For Soluplus ${ }^{\circledR}$ and EPO there was evidence of crystalline IsoNA within the 617 matrices and negligible Ibu/IsoNA cocrystal product. Moreover, for both 618 polymeric systems, there was no evidence within XRD patterns of crystalline Ibu, 619 after extrusion. In the case of Soluplus ${ }^{\circledR}$, the lack of crystalline Ibu may be 620 attributed to heating the drug beyond its melting point during extrusion $\left(92^{\circ} \mathrm{C}\right)$ 621 and entrapping the drug in a highly viscous polymer network. The entrapment of 622 Ibu molecules within rubbery and highly viscous Soluplus ${ }^{\circledR}$ would lead to 623 reduced mobility of $\mathrm{Ibu}$ and consequently reduce the interaction with IsoNA, 624 limiting cocrystal yield. Conversely, when utilising EPO as a matrix carrier, 625 competition between EPO and the coformer isonicotinamide to form hydrogen 626 bonds with ibuprofen would further impact upon cocrystal yield. There have 
627 been many reports of interactions between carboxylic acids and EPO $66-68$. 628 Additionally, we would expect, as with Soluplus ${ }^{\circledR}$, that the mixing of Ibu with 629 highly viscous rubbery EPO would physically hinder molecular interaction 630 between the cocrystal reagents limiting cocrystal yield.

631 Conversely, the small molecular weight sugar alcohol used in this study 632 (xylitol), was found to assist cocrystallization. In part, this may be attributed to 633 limited miscibility between cocrystal product and Xylitol. Indeed, polyols have 634 been shown previously to be relatively inert during extrusion ${ }^{69}$. Furthermore, it 635 is well accepted that low molecular weight solvents (typically volatile organic 636 solvents) can significantly improve cocrystal yield. There are many reported 637 articles describing the significant increase in yield associated with solvent638 assisted-methods relative to neat preparation ${ }^{17,70,71}$. In comparison to Soluplus ${ }^{\circledR}$ 639 and EPO, Xylitol is a low molecular weight (152 g/mol) carrier, with a melt 640 viscosity typically $3 \sim 4$ orders of magnitude lower than the two polymeric 641 excipients. The lack of miscibility between the cocrystal and xylitol and the low 642 viscosity and hence, increased molecular mobility of cocrysal reagents appear to 643 have been key drivers in the successful production of cocrystal. Moreover, the 644 low viscosity, ease by which cocrystal may disperse throughout the melt ${ }^{58}$ and 645 the rapid solidification of xylitol post-extrusion, led to a solid extrudate with 646 cocrystal well dispersed throughout the xylitol matrix.

647 Cocrystals formed using xylitol as a matrix carrier possessed the same 648 hydrogen-bonding pattern (FTIR) and crystal structure (XRD) as the reference 649 cocrystal manufactured via solvent evaporation and the neat-extruded cocrystal . 650 The inert nature of xylitol and the limited miscibility with the cocrytal is 651 fundamental to successful cocrystal formation during extrusion. Strong non652 covalent interactions between any ingredients, other than a coformer pair, may 653 be detrimental to product formation and yield. Therefore, it becomes very 654 important to understand and if necessary quantify the strength of interaction 655 between all components to ensure successful cocrystal formation during 656 extrusion.

657 From this work, it is evident that cocrystal suspensions may be successfully 658 manufactured in a single step using extrusion provided a matrix carrier has 659 specific qualities. Potential matrix carriers should have limited ability to form 
non-covalent interactions with all cocrystal components, a sufficiently low processing temperature such that it is lower than cocrystal melting temperature and rapid solidification upon cooling.

The improvement in the dissolution of drug compounds, when manufactured as cocrystals, can be used to enhance the solubility of BCS class II drugs. It was evident that the addition of small quantities of xylitol (10\%) increased cocryatl yield however at higher xylitol concentrations (30\% and 50\%), the cocrystal yield was equivalent to the formulation devoid of xylitol (Formulation 1). There was no evidence of crystalline ibuprofen immediately after processing, indicating that residual ibuprofen was rendered amorphous. Therefore, the amorphous content (as percentage of the total Ibu content) in the $30 \%$ and $50 \%$ xylitol formulations were equivalent to that in the neat-extruded formulation. And the amorphous Ibu content in the $10 \%$ xylitol formulation was less than that in the system devoid of xylitol.

In drug dissolution studies, formulation 1 (devoid of xylitol) exhibited significantly increased rate and extent of drug release relative to the crystalline ibuprofen powder. This could be the result of a combined effect of the formation of both the cocrystal and amorphous forms of ibuprofen. Although it is difficult to quantify the impact of each individual form to the dissolution improvement, the fact that formulations 1, 5 and 6 all had equivalent amounts of cocrystal yield is suggesting that the further increased dissolution rates of formulations 5 and 6 , relative to formulation 1 , are attributed to the presence of xylitol.

The enhanced dissolution rate observed for a cocrystal suspension embedded in a hydrophilic matrix relative to neat cocrystal powders may be attributed to (1) the cocrystal particles present in the suspension are less aggregated due to the distribution within the matrix carrier, facilitated by agitation caused by extrusion screw rotation and (2) improved wettability owing to the hydrophilicity of the carrier ${ }^{42,72-75}$. For the matrix containing Soluplus ${ }^{\circledR}$, the release of Ibu initiated rapidly due to the presence of amorphous Ibu at the surface of the Soluplus ${ }^{\circledR}$ matrix. However, the erosion of the Soluplus ${ }^{\circledR}$ matrix was significantly slower than that of formulations containing xylitol or those devoid of excipient. With IsoNA rapidly dissolving into the dissolution medium due to its high aqueous solubility, the rapid ingress of water into the Soluplus ${ }^{\circledR}$ 
matrix may have increased Ibu mobility causing clustering of the dispersed Ibu molecules and their subsequent recrystallization within the matrix. Conversely, the formulation containing EPO, exhibited a reduced dissolution rate. This retardation of drug release may be due to the cationic nature of the polymer, the interaction with the acidic drug and the inherent slow dissolution of the polymeric carrier in the chosen dissolution media.

Many approaches have been used to enhance the dissolution performance of BCS class II drugs. Cocrystals have not only been shown to improve drug release properties but are also more physically stable than amorphous drug forms during storage. To understand the storage stability of the extrudates cocrystal suspensions were desiccated under two different conditions, namely, a dry environment over silica gel, and humid condition maintained at $70 \% \mathrm{RH}$, both at $20^{\circ} \mathrm{C}$ for 12 months. As previously discussed, hydrogen bonds between the two cocrystal reagents are stronger than that between the homo-molecules ${ }^{6,41}$, it is therefore of relevance to confirm if the formed cocrystal could stay unchanged under pharmaceutically relevant storage conditions. Indeed, both Raman mapping and PXRD patterns showed that the stored samples had varying degrees of cocrystal growth as a result of aging. This is quantitatively indicative of incomplete cocrystallisation during extrusion. However, both DSC and PXRD analyses on extrudates immediately following manufacture showed little evidence of the presence of crystalline Ibu or IsoNA. The growth of cocrystal during storage may be attributed to interaction between amorphous Ibu and IsoNA molecules, that subsequently form cocrystals ${ }^{76}$. The samples stored under high humidity, in particular, showed significant cocrystal growth during storage most probably due to increased global molecular mobility following ingress of moisture into the xylitol matrix. Consequently, it may be concluded that even if reagents are partially amorphous following extrusion and are physically stabilised by the presence of a matrix carrier they may still recrystallize. This is driven by the ingress of moisture, the drop in $\mathrm{Tg}$, associated increase in molecular mobility of the amorphous components ${ }^{77}$ and the subsequent formation of a thermodynamically favourable cocrystal product.

Furthermore, the incomplete cocrystal conversion may be attributed to the rapid transport of material through the extrusion barrel, limiting reaction time. 
726 The residence time of a typical HME process is a multifactorial-controlled 727 variable that is dependent upon factors such as the properties of the extruded 728 materials, the processing parameter settings and, the machine geometry ${ }^{78-82}$. For 729 larger scale extruders, residence time may be prolonged due to physically 730 extended barrel length. Limitation with respect to limited reaction time may be 731 overcome using increased mixing intensity. An incorrect choice of screw 732 geometry may lead to inadequate mixing in the barrel and reduce cocrystal 733 yield 27,28 . The extruder utilized in this work consisted of a $10 \mathrm{~cm}$ long conical 734 barrel coupled with non-intermeshing conical co-rotating screws. A full 735 conveying screw design was employed throughout the entire length of the 736 barrel. With this set-up, the compression of materials occurs by decreasing the 737 barrel volume in the direction of melt flow. When extruding a cocrystal 738 suspension from a mixture of the reagent pair and a chosen carrier, however, 739 such an extruder design (limited mixing intensity) may not be aggressive enough 740 to compensate for the limited residence time In addition, it may also be 741 important to consider the solubility of cocrystal parent reagents in the carrier. A 742 liquefied carrier that cannot solubilize the parent reagents may reduce 743 interspecies collision. Conversely, carrier-reagent reactivity should be less than 744 reagent-reagent reactivity in order to encourage increased cocrystal yield. 745 Theoretically, the solubility of the components within this process would be 746 expected to be temperature dependent. Thus a detailed investigation into the 747 influence of processing temperature on the solubility between components and 748 hence the cocrystal conversion is necessary.

\section{Conclusion}

751 This work demonstrated the viability of concurrent cocrystallisation and drug 752 product formulation in a miniature scale $(10 \mathrm{~g})$ co-rotating twin-screw extruder. 753 The final extrudates were examined to be intimate mixtures wherein the newly 754 formed cocrystal particulates were physically suspended in a matrix formed by a 755 'inert' carrier excipient. Importantly, it was established in this study that an 756 appropriate carrier for a cocrystal reagent pair during HME processing should 757 satisfy certain criteria including: (1) limited interaction with parent reagents and 758 cocrystal product; (2) processing temperature sufficiently lower than the onset 
759 of cocrystal $\mathrm{T}_{\mathrm{m}}$; (3) low melting viscosity; and (4) fast solidification upon cooling. 760 In conclusion, the use of low viscosity, chemically 'inert' matrix carriers may be 761 successfully employed in the mechanochemical synthesis of pharmaceutical 762 cocrystal suspensions via HME. 


\section{Figures}<smiles>CC(C)Cc1ccc(C(C)C(=O)O)cc1</smiles>

Ibuprofen<smiles>NC(=O)c1cccnc1</smiles>

Nicotinamide<smiles>NC(=O)c1ccncc1</smiles>

Isonicotinamide

(a)

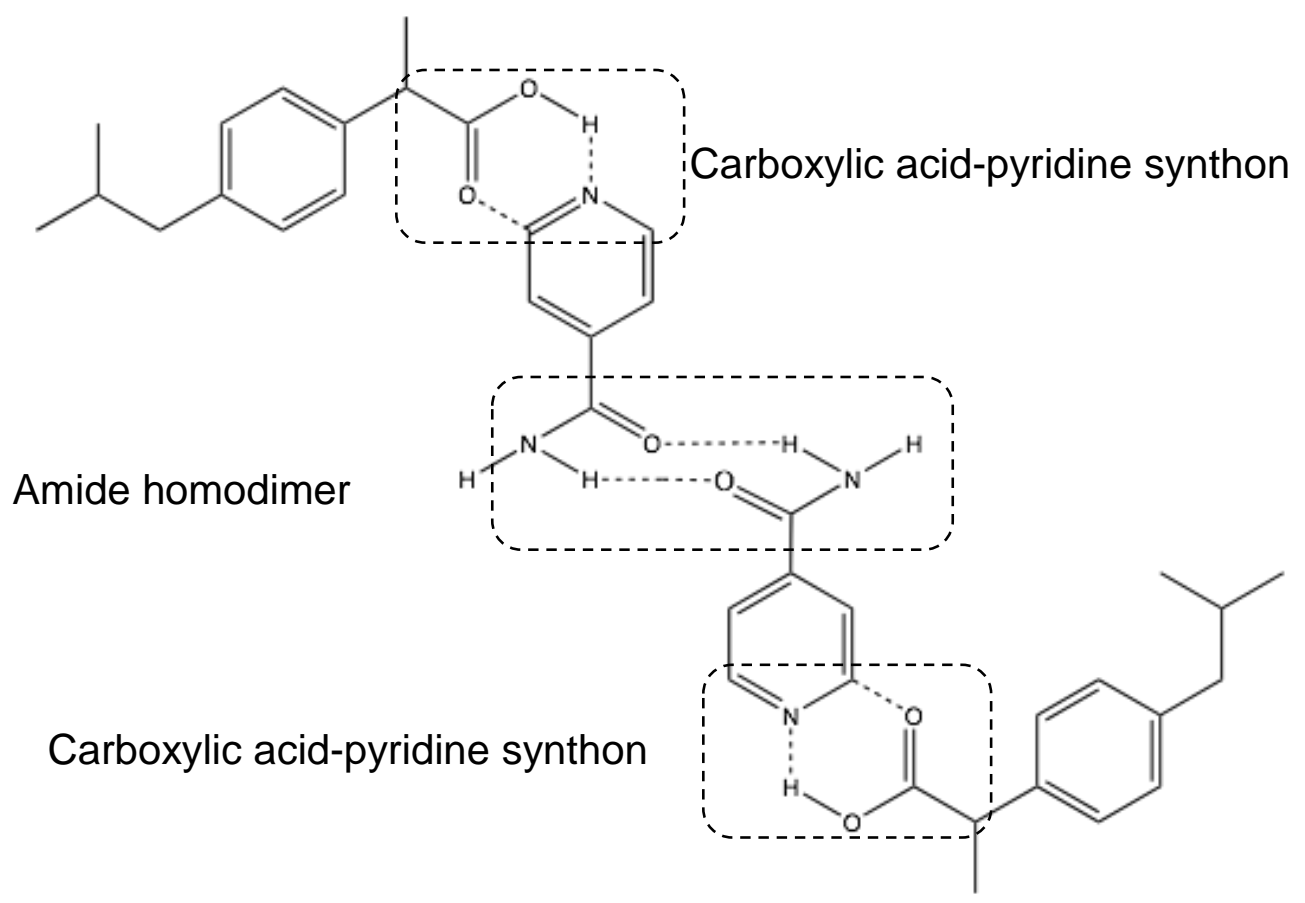

(b)

Figure 1(a). Molecular structure of Ibuprofen, nicotinamide, isonicotinamide and (b) proposed theoretical architecture of an ibuprofen/IsoNA cocrystal (FriŠčić \& Jones 2007, Karki et al; 2007). Hydrogen bonds are shown as dotted lines. 


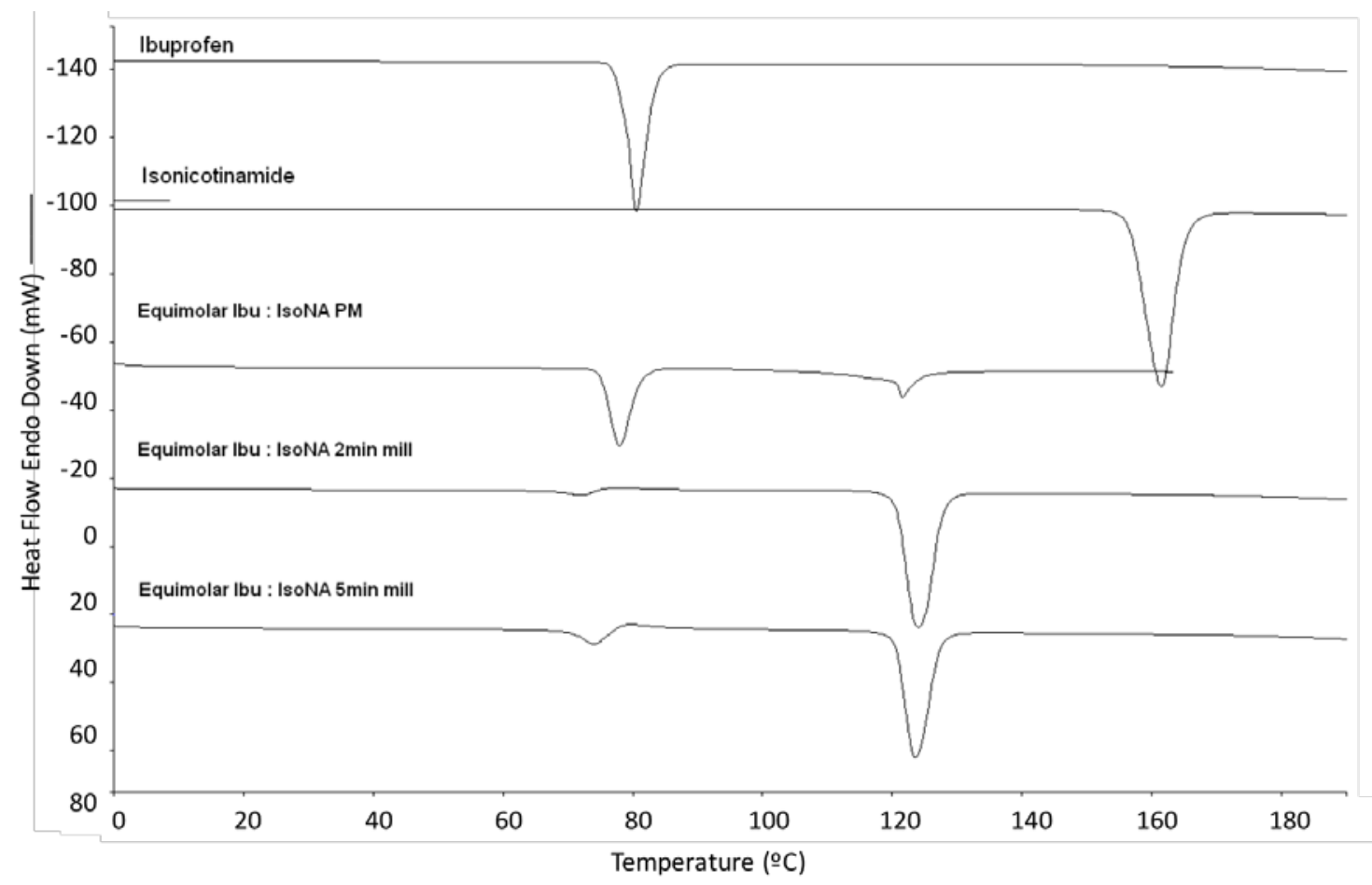

Figure 2 Representative DSC thermogram from top to bottom: crystalline ibuprofen; crystalline isonicotinamide (as is); physical mixture (PM) of ibuprofen \& isonicotinamide at 1:1 molar ratio; equimolar Ibu/IsoNA ball milled for 2 minutes; and equimolar Ibu/IsoNA ball milled for 5 minutes.

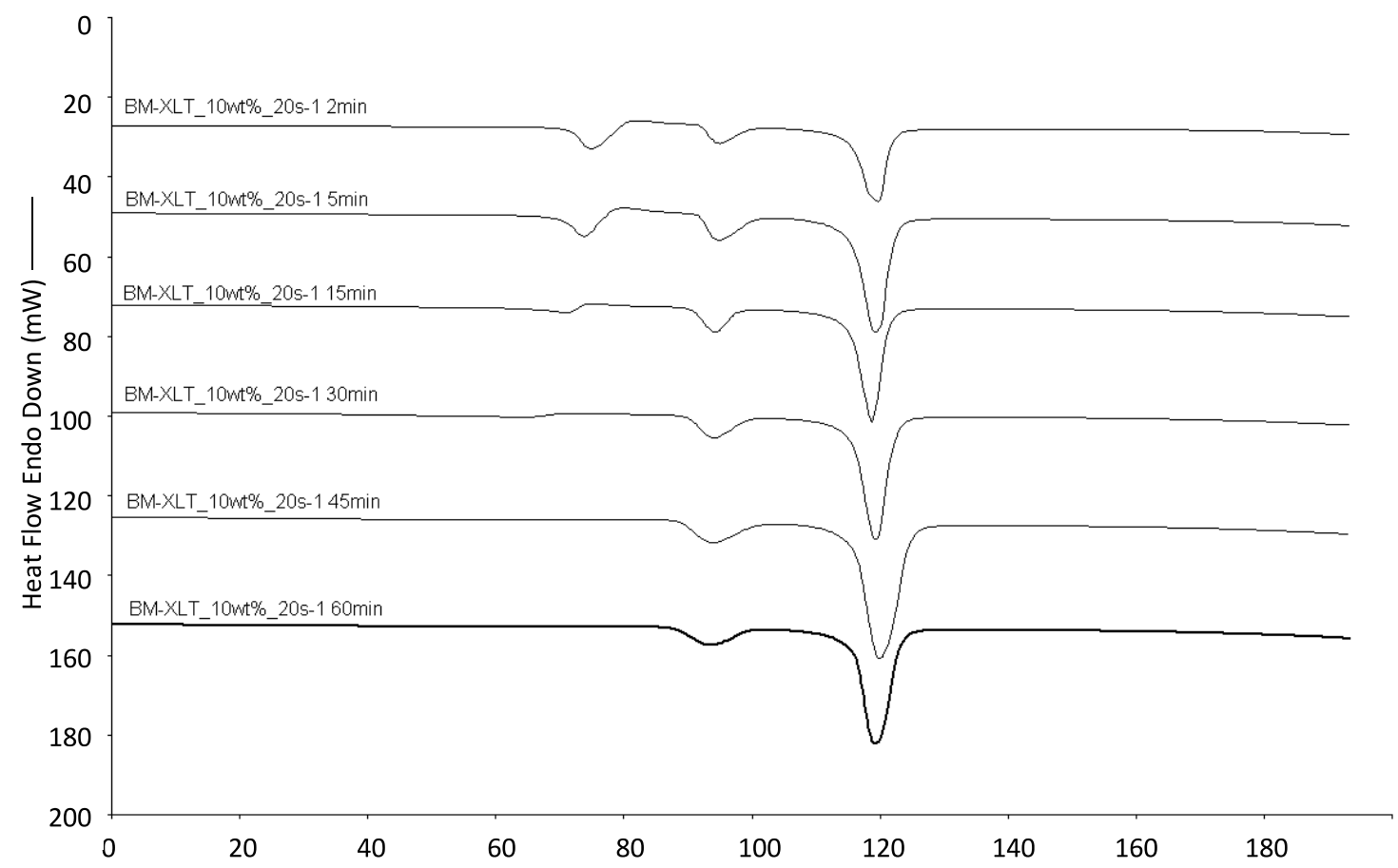

Figure 3 Overlaid DSC thermograms showing the formation and increase of Ibu/IsoNA cocrystal in the presence of $10 \mathrm{wt} \%$ xylitol after (from top to bottom): 2, 5, 15, 30, 45 and, 60 minutes ball milling. 


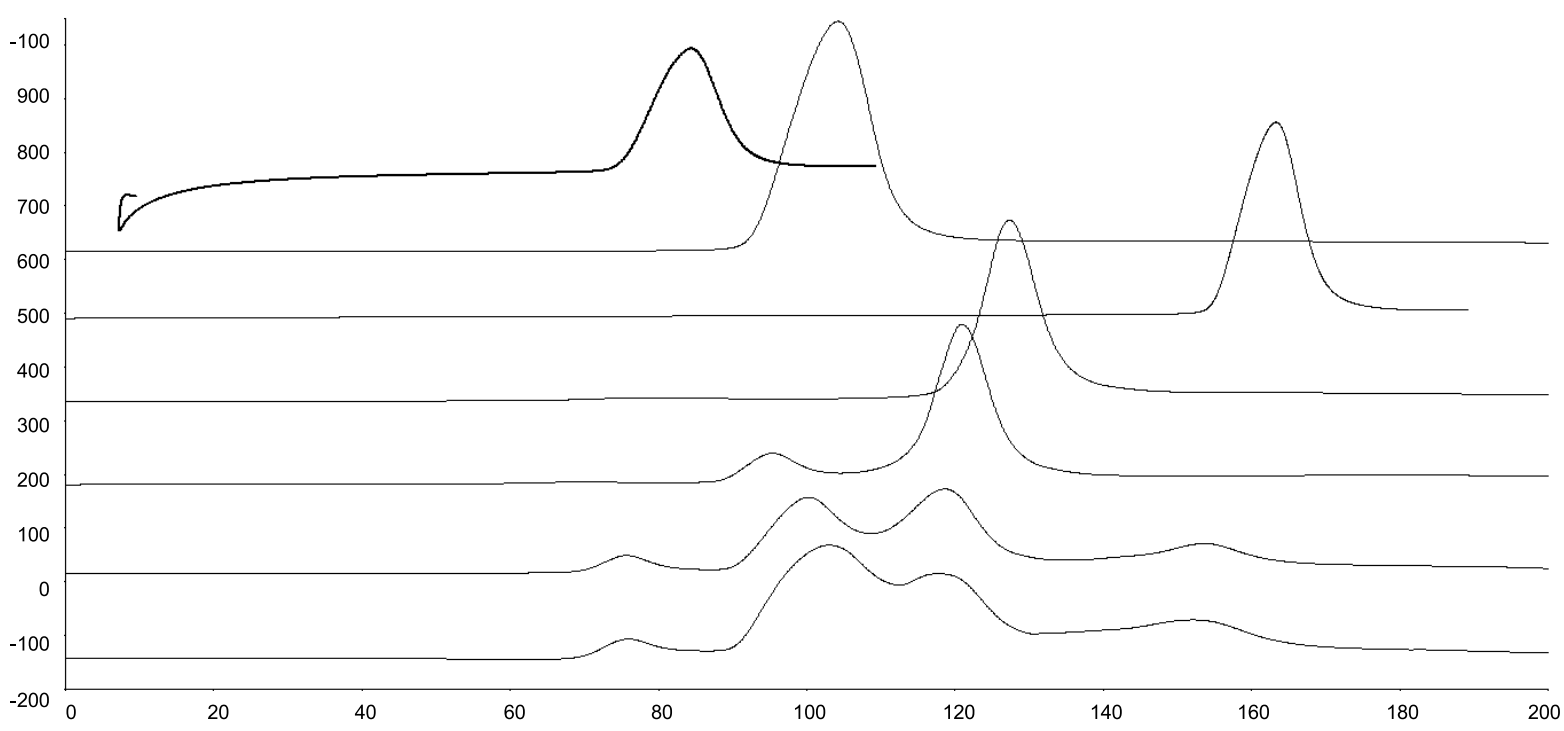

Figure 4 Representative DSC thermograms of materials used in cocrystal formulation development. From top to bottom: ibuprofen, xylitol, isonicotinamide, reference cocrystal standard prepared using solution method, extruded Ibu/IsoNA cocrystal suspension in $10 \mathrm{wt} \%$ xylitol; extruded Ibu/IsoNA cocrystal suspension in 30wt $\%$ xylitol and, extruded $\mathrm{Ibu} / \mathrm{IsoNA}$ cocrystal suspension in $50 \mathrm{wt} \%$ xylitol. 


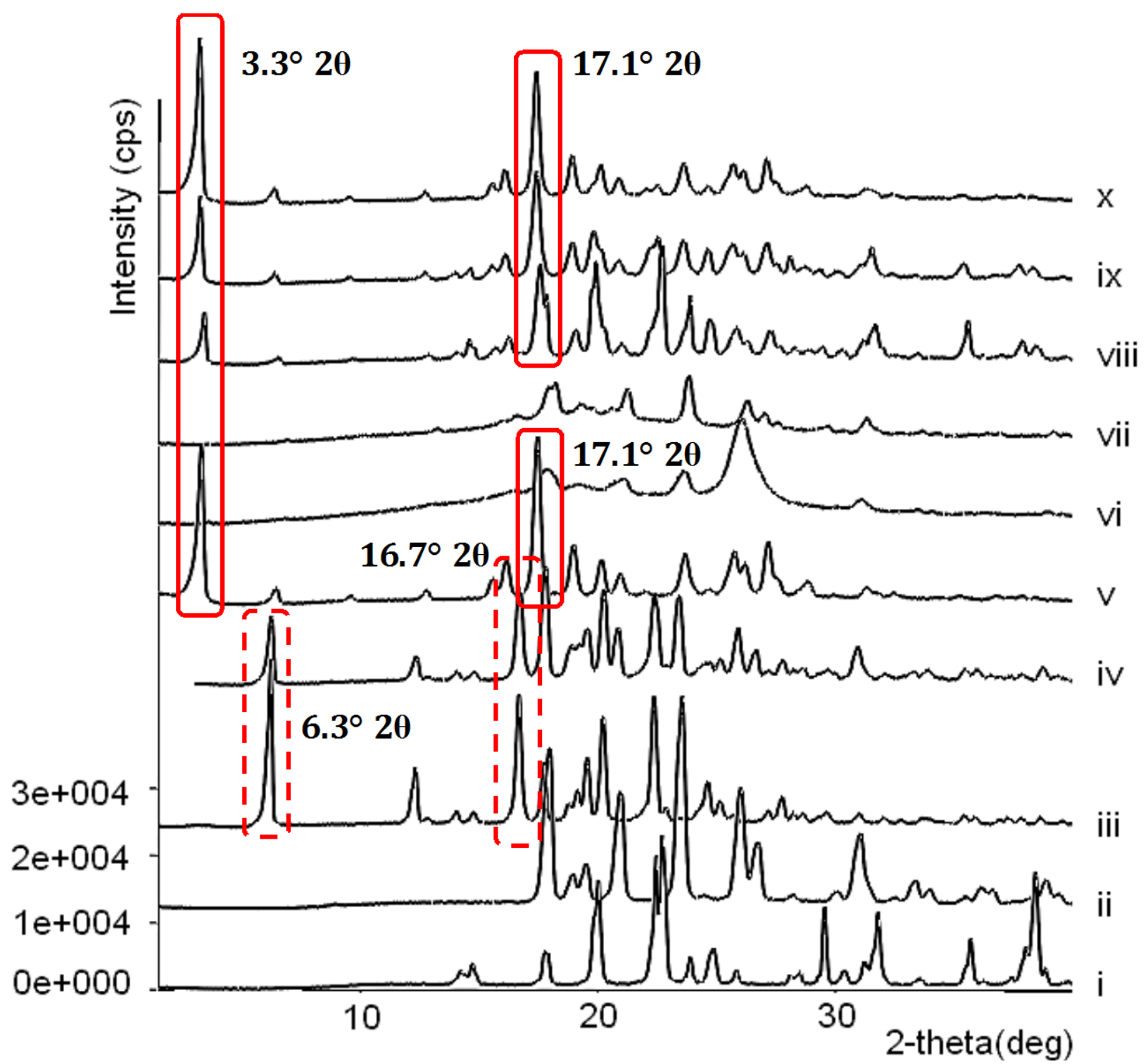

Figure 5 Overlaid PXRD patterns of: (i) xylitol; (ii) IsoNA; (iii) Ibu; (iv) equimolar Ibu/IsoNA physical mitxture; (v) $1: 1$ neat extruded at $92^{\circ} \mathrm{C}$; (vi) $10 \mathrm{wt} \%$ Soluplus ${ }^{\circledR}$ HME; (vii) 10wt\% EPO HME; (viii) 50wt\% xylitol HME; (ix) 30wt\% xylitol HME; and (x) 10wt\% xylitol HME. Please note: all extrudates contained ibuprofen and isonicotinamide at a 1:1 molar ratio. 


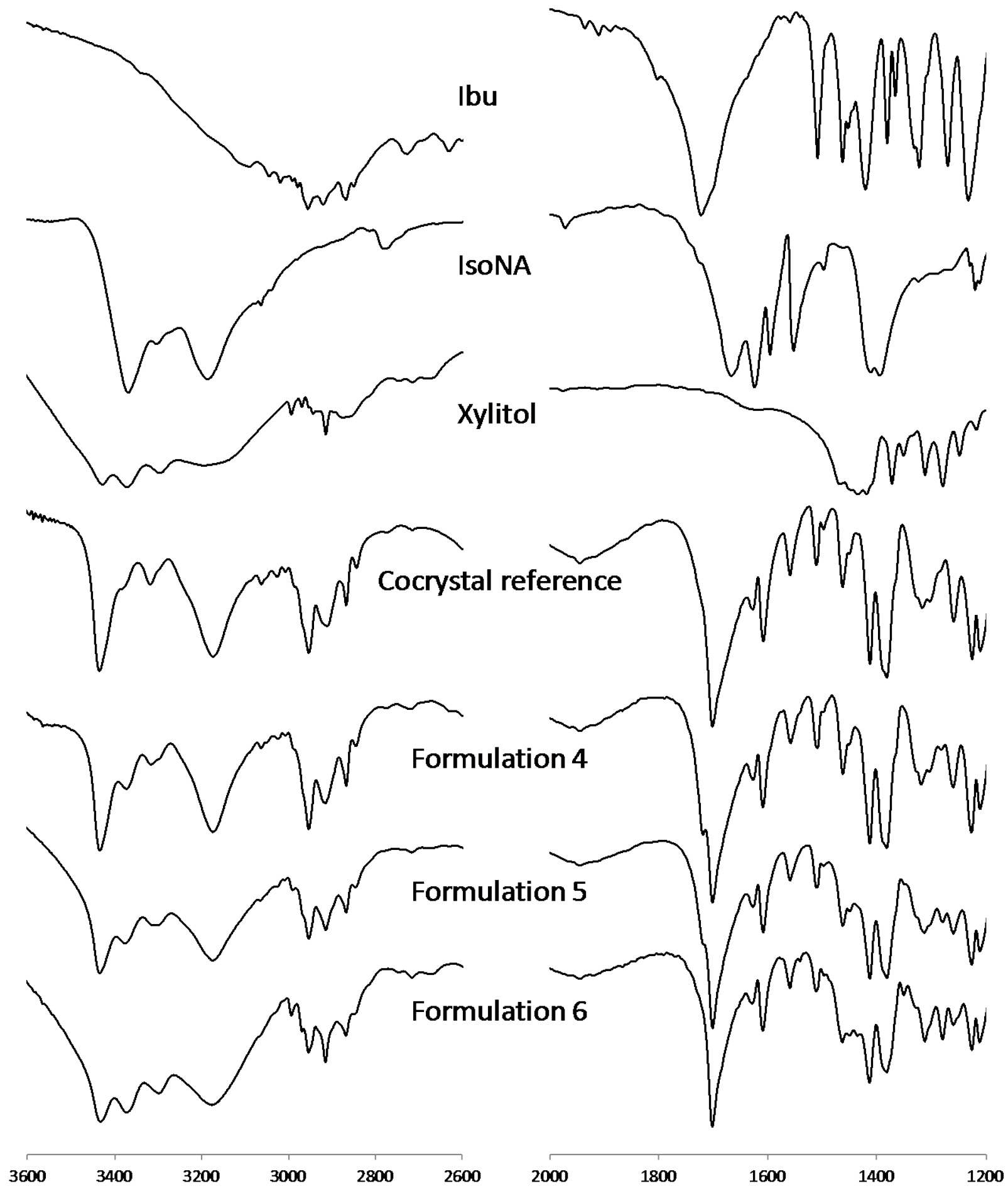

Figure 6 Overlaid FTIR spectra within wavenumber ranges of [2600-3600 $\left.\mathrm{cm}^{-1}\right]$ and [1200$2000 \mathrm{~cm}^{-1}$ ], respectively for, from top to bottom: Ibu, IsoNA, xylitol, the cocrystal reference, extruded suspensions containing 10\%, 30\% and, 50\% xylitol, respectively. 


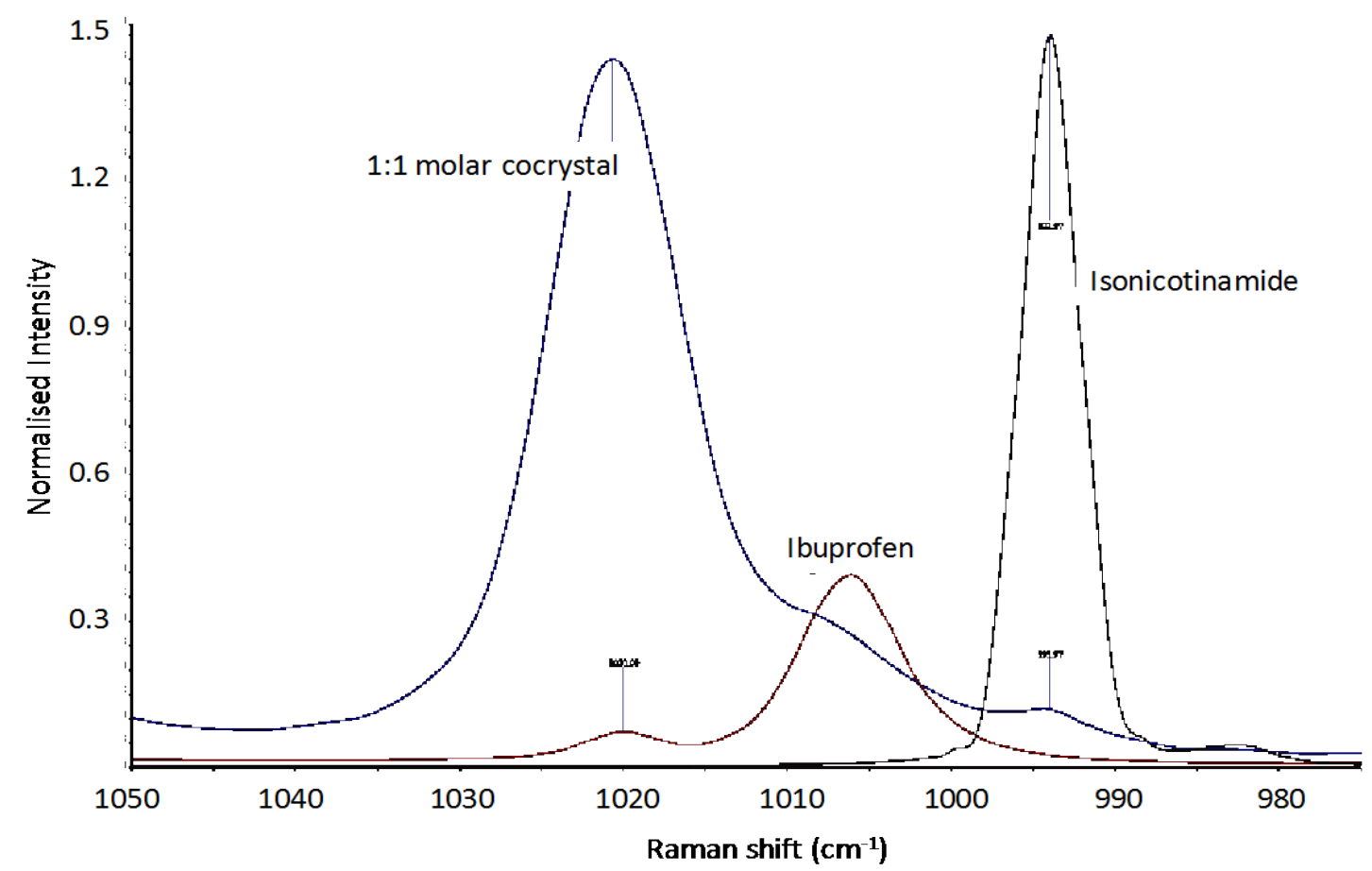

Figure 7 Raman shift region [1050.0 975.0 cm-1] showing non-interfering, characteristic peaks for: (Red) unprocessed Ibuprofen; (green) unprocessed IsoNA; and (blue) the equimolar cocrystal prepared using slow evaporation.

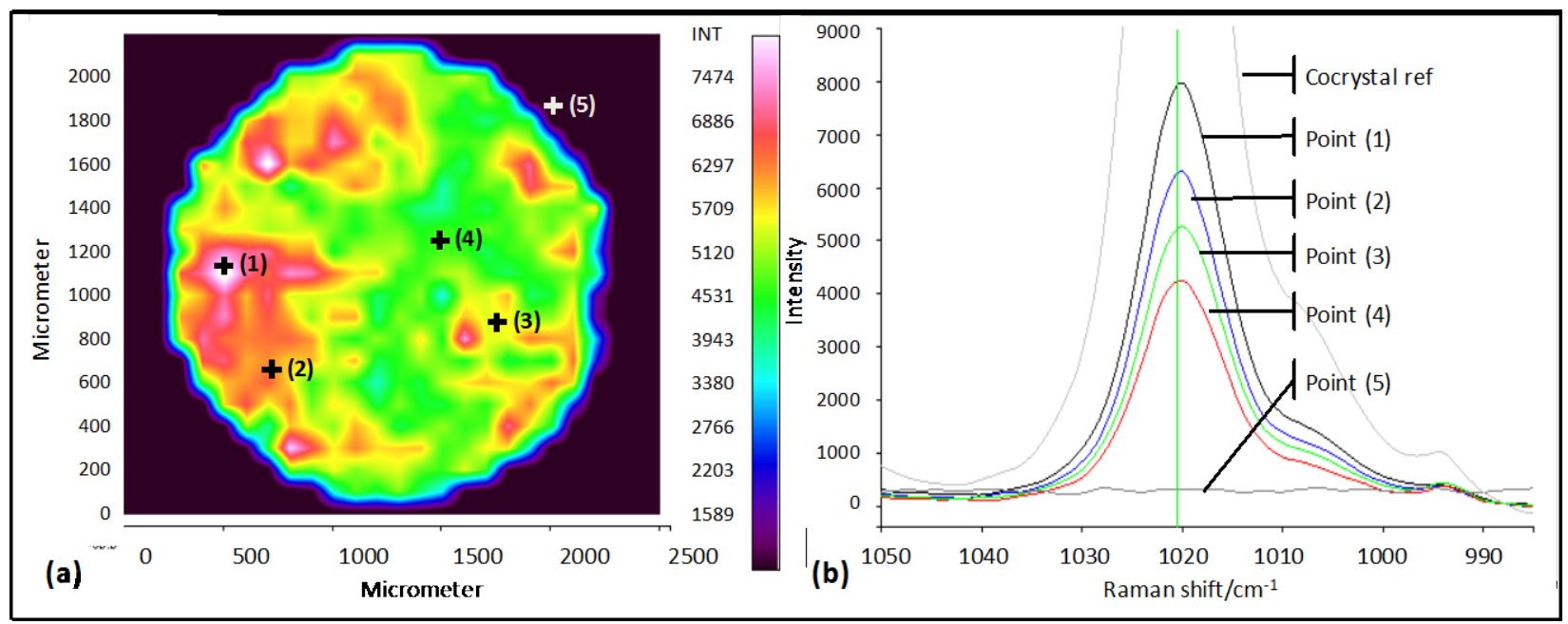

Figure 8 (a) Schematic demonstration of the occurrence and concentration of the cocrystal across the cross section of a formulation 1 extrudate. (b) The spectra showing the intensity of the cocrystal peak at $1020 \mathrm{~cm}^{-1}$ at the labelled points and that of the cocrystal reference are shown in the right figure in an overlaid format. 


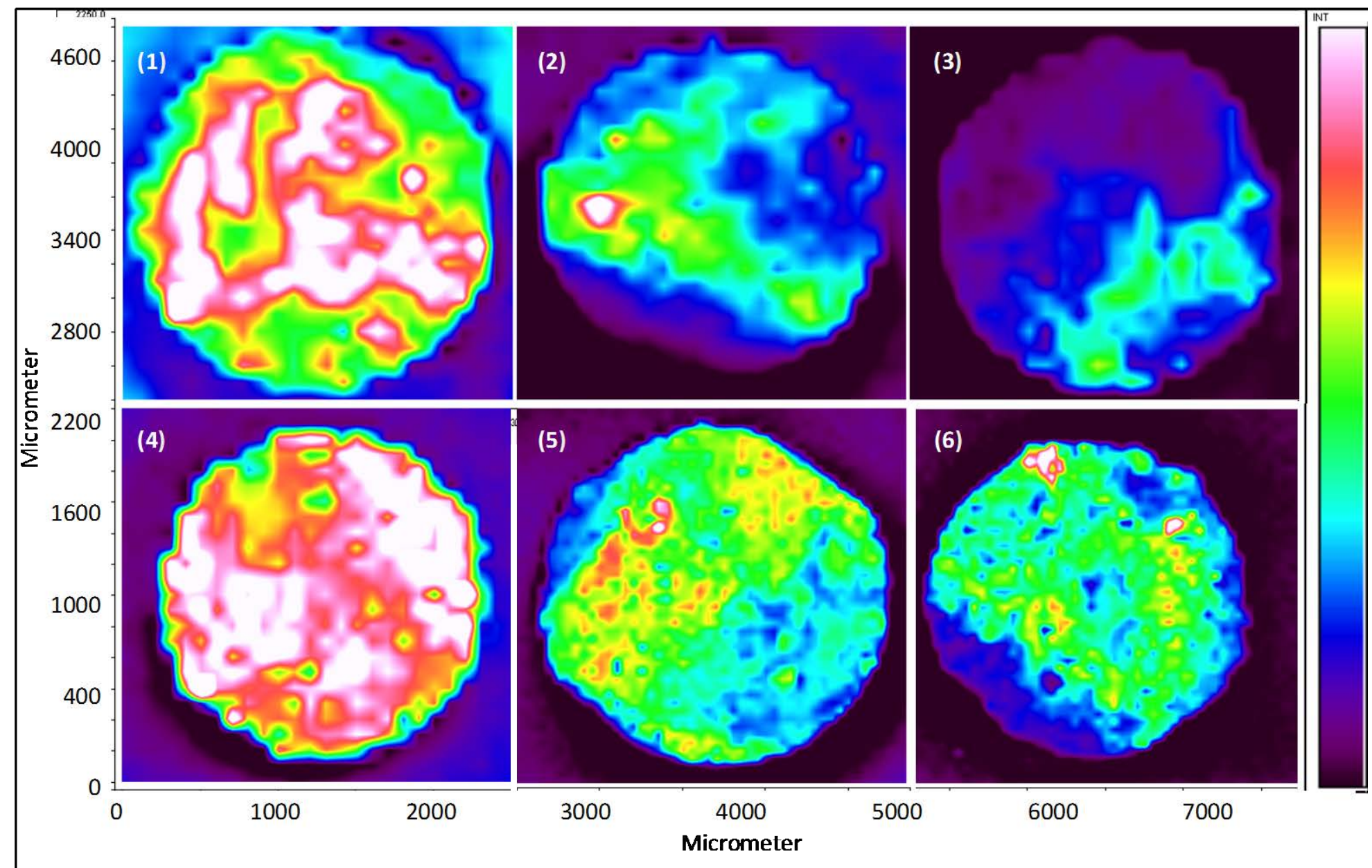

Figure 9 Raman map/image showing the intensity of the peak at $1020 \mathrm{~cm}^{-1}$, characteristic of the cocrystal, throughout the cross section of: (1) fresh extrudates of formulation 4, containing 10\% xylitol; (2) fresh extrudates of formulation 5, containing 30\% xylitol; (3) fresh extrudates of formulation 6, containing 50\% xylitol; (4) aged formulation 4; (5) aged formulation 5; and (6) aged formulation 6. 

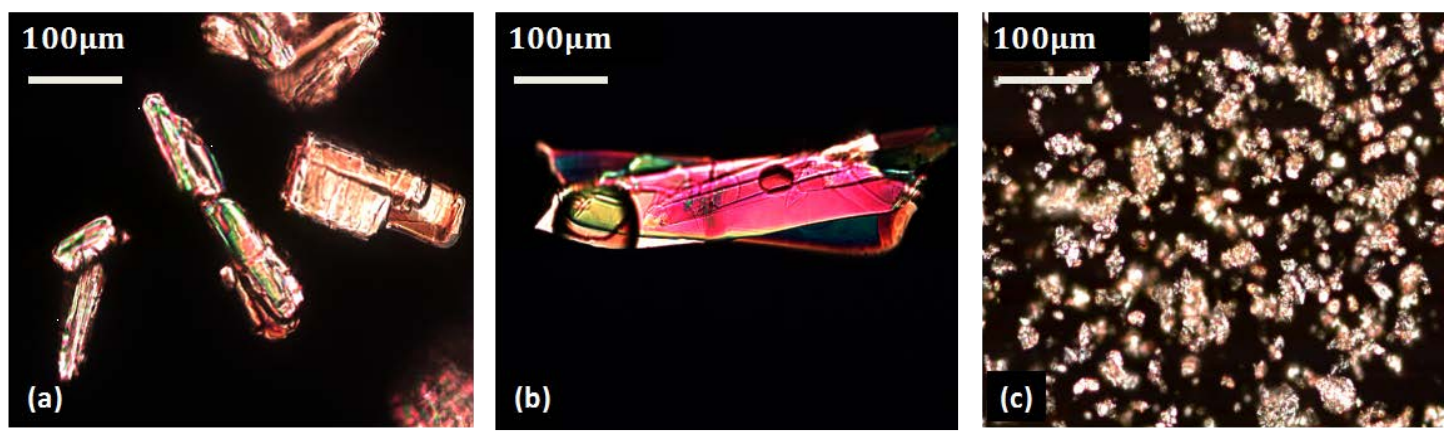

Figure 10 Polarized light micrographs showing crystal habit and size of: (a) unprocessed Ibu; (b) reference 1:1 Ibu/IsoNA cocrystal prepared using solvent evaporation; and (c) melt-extruded cocrystal particles. Particulates chosen all passed through $212 \mu \mathrm{m}$ sieve and were dispersed in mineral oil $(200 \mathrm{x}$, the entire width of each picture represents $0.5 \mathrm{~mm}$ on a magnified scale bar). 


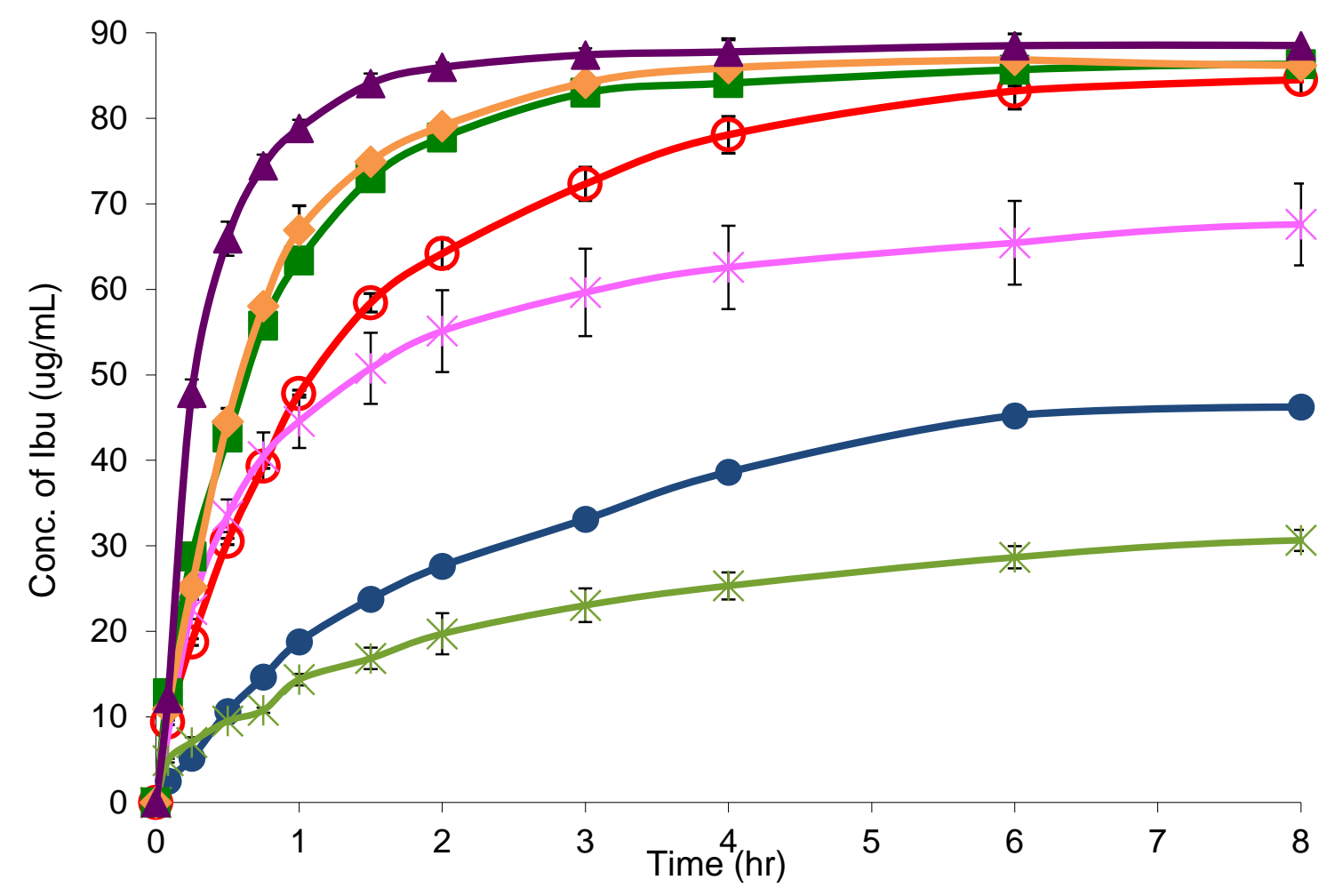

Figure 11 Drug dissolution profiles of melt extruded equimolar Ibu/IsoNA formulations in deionized water. Profiles from bottom to top: (*) extrudates containing Eudragit ${ }^{\circledR}$ EPO; ( $\bullet$ ) Pure ibuprofen powders; (*) extrudates containing Soluplus ${ }^{\circledR}$; (O) 1:1 melt-extruded cocrystal (formulation 1); ( $\square$ ) 1:1 extruded cocrystal suspension in 10\% xylitol (formulation 4); ( $\vee$ ) 1:1 extruded cocrystal suspension in 30\% xylitol (formulation 5); and (४) 1:1 extruded cocrystal suspension in $50 \%$ xylitol (formulation 6). Each point represents the mean \pm S.D. of 3 replicates. 


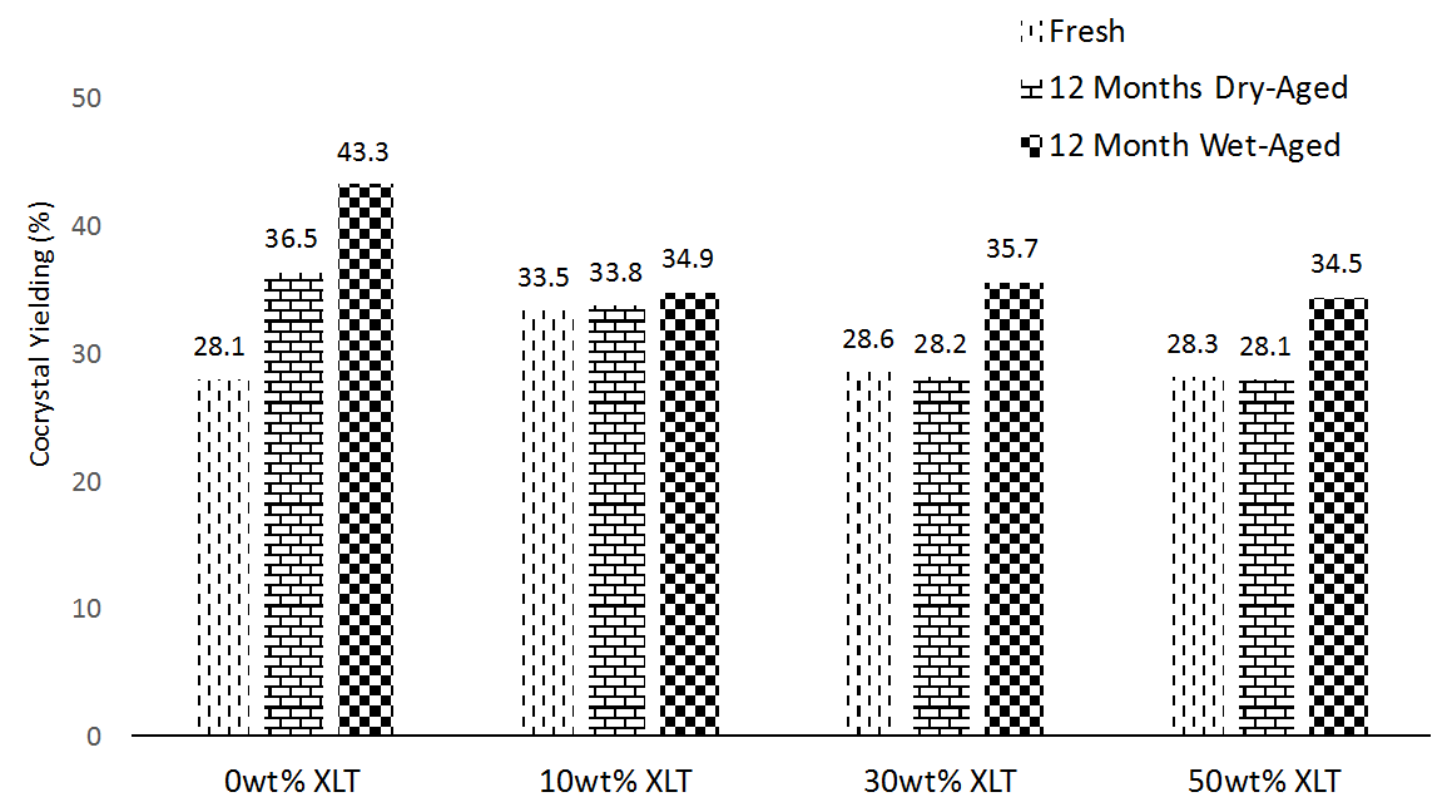

Figure 12 Yielded cocrystal content determination for freshly extruded and stored cocrystal suspension formulations containing (from left to right): $0 \%$, $10 \%, 30 \%$ and, 50\%, w/w xylitol. 


\section{Tables}

Table 1 Nomenclature and the Parameter Settings for the Hot-Melt Extruded Formulations Composed of Equimolar Ibu and IsoNA, as well as a Third Matrix Carrier. a

\begin{tabular}{|c|c|c|c|c|c|c|c|c|c|c|}
\hline Formula & $\begin{array}{c}\text { Ibu } \\
\text { wt\% }\end{array}$ & $\begin{array}{c}\text { IsoNA } \\
\mathrm{wt} \%\end{array}$ & $\begin{array}{c}\text { Soluplus }{ }^{\circledR} \\
\text { wt } \%\end{array}$ & $\begin{array}{c}\text { Eudragit }^{\circledR} \\
\text { EPO } \\
w t \%\end{array}$ & $\begin{array}{c}\text { Xylitol } \\
\text { wt\% }\end{array}$ & $\begin{array}{c}\text { Feed \& Mix } \\
\text { Temp/speed } \\
{ }^{\circ} \mathrm{C} / \mathrm{rpm}\end{array}$ & $\begin{array}{c}\text { Flushing } \\
\text { Temp/speed } \\
{ }^{\circ} \mathrm{C} / \mathrm{rpm}\end{array}$ & $\begin{array}{c}\text { Residence } \\
\text { time } \\
\mathrm{s}\end{array}$ & $\begin{array}{c}\text { Flush } \\
\text { Torque } \\
\text { Ncm }\end{array}$ & Outcome \\
\hline 1 & 62.82 & 37.18 & - & - & - & $92 / 10$ & $92 / 10$ & 233 & $40 \sim 44$ & Fragile rods \\
\hline 2 & 56.54 & 33.46 & 10 & - & - & $92 / 10$ & $92 / 10$ & 241 & $4 \sim 6$ & Sticky strand \\
\hline 3 & 56.54 & 33.46 & - & 10 & - & $92 / 10$ & $92 / 10$ & 220 & $4 \sim 6$ & Sticky strand \\
\hline 4 & 56.54 & 33.46 & - & - & 10 & $92 / 10$ & $85 / 50$ & 90 & $109 \sim 122$ & Brittle strand \\
\hline 5 & 43.98 & 26.02 & - & - & 30 & $92 / 10$ & $85 / 50$ & 272 & $43 \sim 59$ & Brittle strand \\
\hline 6 & 31.41 & 18.59 & - & - & 50 & $92 / 10$ & $85 / 50$ & 329 & $19 \sim 22$ & Brittle strand \\
\hline
\end{tabular}

${ }^{a}$ Note that the weight ratios tabulated here provide 1:1 molar ratio for Ibu and IsoNA in the blends. The batch size was maintained at approximately $10 \mathrm{~g}$ for each formulation and the extrudates were collected after equilibration for 5 minutes. 
Table 2. The Molecular Weights, Melting/Glass Transition Temperatures and Decomposition Temperatures of Each Individual Compound used in This Study. a

\begin{tabular}{l|ccc}
\hline Compound & Mw & Tm or $\mathbf{~ T g}$ & $\mathbf{T}_{5 \text { wt } \% \text { loss }}$ \\
& $\mathrm{g} / \mathrm{mol}$ & ${ }^{\circ} \mathrm{C}$ & ${ }^{\circ} \mathrm{C}$ \\
\hline Ibu & 206.30 & $84.41 \pm 0.57$ & $197.44 \pm 3.67$ \\
IsoNA & 122.12 & $163.26 \pm 0.99$ & $188.10 \pm 2.14$ \\
$\begin{array}{l}\text { Ibu/IsoNA } \\
\text { cocrystal }\end{array}$ & 656.48 & $127.29 \pm 0.55$ & $164.07 \pm 6.77$ \\
Xylitol & 152.15 & $104.17 \pm 0.29$ & $270.03 \pm 0.43$ \\
Eudragit ${ }^{\circledR}$ E PO & 47,000 & $43.94 \pm 0.13$ & $279.70 \pm 1.00$ \\
Soluplus ${ }^{\circledR}$ & $90,000 \sim 140,000$ & $66.52 \pm 0.20$ & $308.57 \pm 0.91$ \\
\hline
\end{tabular}

a The temperatures shown here represent the mean \pm SD of three replicates. Note that the values of $\mathrm{T}_{\mathrm{m}}$ listed the peak maximums measured at $200^{\circ} \mathrm{C} / \mathrm{min}$. 
Table 3 Assignment for the Most Characteristic Vibrational Bands of Ibu and IsoNA in the Raw Materials and the 1:1 Melt-Extruded Ibu/IsoNA Cocrystal.

\begin{tabular}{|c|c|c|c|}
\hline & $\begin{array}{l}\text { IR Frequency } \\
\left(\mathrm{cm}^{-1}\right)\end{array}$ & $\begin{array}{l}\text { Raman Shift } \\
\left(\mathrm{cm}^{-1}\right)\end{array}$ & Band assignment a \\
\hline \multirow{4}{*}{ Ibuprofen } & $3400 \sim 2800$ & - & vo-H (Associated) \\
\hline & 1721.16 (vs) & 1605.95 & $\nu_{\mathrm{C}=0}($ Carboxylic acid $)$ \\
\hline & $1007.62(\mathrm{~m})$ & 1006.13 & $v_{\text {c-c }}($ Aromatic ring chain vib) \\
\hline & $796.457(\mathrm{~m})$ & - & $\gamma=\mathrm{C}-\mathrm{H}($ Aromatic ring $)$ \\
\hline \multirow{6}{*}{ Isonicotinamide } & 3369.03 (vs) & 3070.09 & $v_{\mathrm{N}-\mathrm{H}}$ (Asymmetric stretching) \\
\hline & 3185.83 (vs) & 3063.81 & $\mathrm{vN}-\mathrm{H}_{\text {(Symmetric stretching) }}$ \\
\hline & 1668.12 (vs) & 1601.58 & $v_{\mathrm{C}=0}($ Stretching $)$ \\
\hline & $1622.80(\mathrm{~s})$ & - & $\delta_{\mathrm{N}-\mathrm{H}}(\mathrm{H}-\mathrm{bonded}$ amide bending) \\
\hline & $1594.84(\mathrm{~m})$ & - & $\delta_{\mathrm{N}-\mathrm{H}}($ Free amide bending) \\
\hline & $1551.45(\mathrm{~m})$ & 993.94 & $\nu_{\text {Ring }}$ (Pyridine ring stretching) \\
\hline \multirow{8}{*}{$\begin{array}{l}\text { Equimolar } \\
\text { Ibu/IsoNA } \\
\text { reference } \\
\text { cocrystal }\end{array}$} & 3434.60 (vs) & - & $v_{\mathrm{N}-\mathrm{H}}($ Free amide $)$ \\
\hline & $3317.93(w)$ & - & $v_{\mathrm{N}-\mathrm{H}}(\mathrm{H}-$ bonded pyridine $\mathrm{N})$ \\
\hline & 3174.26 (vs) & - & $v_{\mathrm{N}-\mathrm{H}}(\mathrm{H}$-bonded amide $)$ \\
\hline & $1702.84(\mathrm{vs})$ & 1612.66 & $v_{\mathrm{C}=0}($ Carboxylic acid $)$ \\
\hline & $1629.55(\mathrm{~m})$ & - & $v_{\mathrm{C}=\mathrm{O}}(\mathrm{H}$-bonded amide $\mathrm{C}=\mathrm{O})$ \\
\hline & $1609.31(\mathrm{~s})$ & - & $\delta_{\mathrm{N}-\mathrm{H}}($ Free amide $)$ \\
\hline & $1560.13(\mathrm{~m})$ & 1020.70 & VRing (Pyridine) \\
\hline & $779.101(\mathrm{~m})$ & - & $\gamma=\mathrm{C}-\mathrm{H}(\mathrm{H}-$ bonded pyridine $=\mathrm{C}-\mathrm{H})$ \\
\hline
\end{tabular}

${ }^{\mathrm{a}} \nu=$ stretching vibration; $\delta=$ in-plane bending; $\gamma=$ out-plane bending. 
Table 4 Dissolution Parameters Calculated for Fig 11.

\begin{tabular}{|c|c|c|c|c|c|c|}
\hline \multirow{2}{*}{ Formulation } & \multicolumn{6}{|c|}{ Dissolution Parameters } \\
\hline & $\mathrm{DP}_{5 \min ^{\mathrm{a}}}$ & $\mathrm{DP}_{45 \mathrm{~min}^{\mathrm{a}}}$ & $\mathrm{DP}_{180 \mathrm{~min}^{\mathrm{a}}}$ & $\mathrm{RDr}_{5 \min }{ }^{\mathrm{b}}$ & $\mathrm{RDr}_{45 \min ^{\mathrm{b}}}$ & $\mathrm{RDr}_{180 \min ^{\mathrm{b}}}$ \\
\hline - Pure Ibu & $1.26 \pm 0.17 \%$ & $7.35 \pm 0.12 \%$ & $16.65 \pm 0.03 \%$ & $0.25 \pm 0.03$ & $0.16 \pm 0.00$ & $0.09 \pm 0.00$ \\
\hline 01 & $4.69 \pm 0.19 \%$ & $19.71 \pm 0.06 \%$ & $36.30 \pm 1.33 \%$ & $0.94 \pm 0.04$ & $0.44 \pm 0.00$ & $0.20 \pm 0.01$ \\
\hline * 2 & $4.73 \pm 0.32 \%$ & $19.83 \pm 1.71 \%$ & $29.08 \pm 3.13 \%$ & $0.95 \pm 0.06$ & $0.44 \pm 0.04$ & $0.16 \pm 0.02$ \\
\hline * 3 & $2.45 \pm 0.10 \%$ & $5.47 \pm 0.08 \%$ & $11.64 \pm 1.36 \%$ & $0.49 \pm 0.02$ & $0.12 \pm 0.00$ & $0.06 \pm 0.01$ \\
\hline च 4 & $5.09 \pm 0.70 \%$ & $27.88 \pm 0.06 \%$ & $41.90 \pm 0.04 \%$ & $1.02 \pm 0.14$ & $0.62 \pm 0.00$ & $0.23 \pm 0.00$ \\
\hline$>5$ & $5.40 \pm 0.08 \%$ & $28.74 \pm 0.18 \%$ & $41.55 \pm 0.03 \%$ & $1.08 \pm 0.02$ & $0.64 \pm 0.00$ & $0.23 \pm 0.00$ \\
\hline$\Delta 6$ & $6.41 \pm 0.07 \%$ & $37.41 \pm 0.81 \%$ & $43.53 \pm 0.34 \%$ & $1.28 \pm 0.01$ & $0.83 \pm 0.02$ & $0.24 \pm 0.00$ \\
\hline
\end{tabular}

a DP: Drug percent (\%) released at a particular time point;

$\mathrm{b}$ RDr: Relative dissolution rate (\%/ minutes) at a particular time point. (RDr=DP/dissolution time). 


\section{References}

(1) McNamara, D.; Childs, S.; Giordano, J.; Iarriccio, A.; Cassidy, J.; Shet, M.; Mannion, R.; O’Donnell, E.; Park, A. Use of a Glutaric Acid Cocrystal to Improve Oral Bioavailability of a Low Solubility API. Pharm. Res. 2006, 23 (8), 1888-1897.

(2) Blagden, N.; de Matas, M.; Gavan, P. T.; York, P. Crystal Engineering of Active Pharmaceutical Ingredients to Improve Solubility and Dissolution Rates. Drug Solubility How to Meas. it, How to Improv. it 2007, 59 (7), 617630.

(3) Jung, M.-S.; Kim, J.-S.; Kim, M.-S.; Alhalaweh, A.; Cho, W.; Hwang, S.-J.; Velaga, S. P. Bioavailability of Indomethacin-Saccharin Cocrystals. J. Pharm. Pharmacol. 2010, 62 (11), 1560-1568.

(4) Mohammad, M. A.; Alhalaweh, A.; Velaga, S. P. Hansen Solubility Parameters as a Tool to Predict Cocrystal Formation. Int. J. Pharm. 2011, 407, 63-71.

(5) Qiao, N.; Li, M.; Schlindwein, W.; Malek, N.; Davies, A.; Trappitt, G. Pharmaceutical Cocrystals: An Overview. Int. J. Pharm. 2011, 419, 1-11.

(6) Vishweshwar, P.; McMahon, J. A.; Bis, J. A.; Zaworotko, M. J. Pharmaceutical Co-Crystals. J. Pharm. Sci. 2006, 95 (3), 499-516.

(7) Thakuria, R.; Delori, A.; Jones, W.; Lipert, M. P.; Roy, L.; RodríguezHornedo, N. Pharmaceutical Cocrystals and Poorly Soluble Drugs. Int. J. Pharm. 2013, 453 (1), 101-125.

(8) Basavoju, S.; Boström, D.; Velaga, S. Indomethacin-Saccharin Cocrystal: Design, Synthesis and Preliminary Pharmaceutical Characterization. Pharmaceutical Research. Springer Netherlands 2008, pp 530-541.

(9) Leyssens, T.; Tumanova, N.; Robeyns, K.; Candoni, N.; Veesler, S. Solution Cocrystallization, an Effective Tool to Explore the Variety of Cocrystal Systems: Caffeine/dicarboxylic Acid Cocrystals. Cryst. Eng. Commun. 2014, 16, 9603-9611.

(10) Trask, A. V.; Jones, W. Crystal Engineering of Organic Cocrystals by the Solid-State Grinding Approach. Topics in Current Chemistry. 2005, pp 41-70.

(11) James, S. L.; Adams, C. J.; Bolm, C.; Braga, D.; Collier, P.; Friscic, T.; Grepioni, F.; Harris, K. D. M.; Hyett, G.; Jones, W.; Krebs, A.; Mack, J.; Maini, L.; Orpen, A. G.; Parkin, I. P.; Shearouse, W. C.; Steed, J. W.; Waddell, D. C.; Friščić, T.; Grepioni, F.; Harris, K. D. M.; Hyett, G.; Jones, W.; Krebs, A.; Mack, J.; Maini, L.; Orpen, A. G.; Parkin, I. P.; Shearouse, W. C.; Steed, J. W.; Waddell, D. C. Mechanochemistry: Opportunities for New and Cleaner Synthesis. Chem. Soc. Rev. 2012, 41 (1), 413-447.

(12) Delori, A.; Friscic, T.; Jones, W.; Friščić, T.; Jones, W. The Role of Mechanochemistry and Supramolecular Design in the Development of Pharmaceutical Materials. CrystEngComm 2012, 14 (7), 2350-2362.

(13) Jones, W.; Eddleston, M. D. Introductory Lecture: Mechanochemistry, a Versatile Synthesis Strategy for New Materials. Faraday Discuss. 2014, 170 (0), 9-34.

(14) Boldyrev, V. V. Mechanochemistry and Mechanical Activation of Solids. Russ. Chem. Rev. 2006, 75 (3), 177-189.

(15) Braga, D.; Grepioni, F. Solventless Reactions: Reactions between or within Molecular Crystals. Angew. Chemie, Int. Ed. 2004, 43 (31), 4002-4011.

(16) Shan, N.; Toda, F.; Jones, W. Mechanochemistry and Co-Crystal Formation: Effect of Solvent on Reaction Kinetics. Chem. Commun. 2002, No. 20, 23722373. 
(17) Trask, A. V; Motherwell, W. D. S.; Jones, W. Solvent-Drop Grinding: Green Polymorph Control of Cocrystallisation. Chem. Commun. 2004, No. 7, 890891.

(18) FriŠčić, T.; Fabian, L.; Burley, J. C.; Jones, W.; Motherwell, W. D. S. Exploring Cocrystal-Cocrystal Reactivity via Liquid-Assisted Grinding: Assembling of Racemic and Dismantling of Enantiomeric Cocrystals . Chem. Commun. 2006, 28 (48), 5009-5011.

(19) FriŠčić, T.; Trask, A. V; Jones, W.; Motherwell, W. D. S. Screening for Inclusion Compounds and Systematic Construction of Three-Component Solids by Liquid-Assisted Grinding. Angew. Chemie 2006, 118 (45), 77087712.

(20) FriŠčić, T.; Childs, S. L.; Rizvi, S. A. A.; Jones, W. The Role of Solvent in Mechanochemical and Sonochemical Cocrystal Formation: A Solubility-Based Approach for Predicting Cocrystallisationoutcome. Cryst. Growth Des. 2009, 11 (3), 418-426.

(21) Yamamoto, K.; Tsutsumi, S.; Ikeda, Y. Establishment of Cocrystal Cocktail Grinding Method for Rational Screening of Pharmaceutical Cocrystals. Int. J. Pharm. 2012, 437 (1-2), 162-171.

(22) Shevchenko, A.; Miroshnyk, I.; Pietilä, L.-O.; Haarala, J.; Salmia, J.; Sinervo, K.; Mirza, S.; van Veen, B.; Kolehmainen, E.; Yliruusi, J. Diversity in Itraconazole Cocrystals with Aliphatic Dicarboxylic Acids of Varying Chain Length . Cryst. Growth Des. 2013, 13 (11), 4877-4884.

(23) Lin, Y.; Yang, H.; Yang, C.; Wang, J. Preparation, Characterization, and Evaluation of Dipfluzine-Benzoic Acid Co-Crystals with Improved Physicochemical Properties. Pharm. Res. 2013, 1-13.

(24) Sowa, M.; Ślepokura, K.; Matczak-Jon, E. A 1:1 Pharmaceutical Cocrystal of Myricetin in Combination with Uncommon Piracetam Conformer: X-Ray Single Crystal Analysis and Mechanochemical Synthesis. J. Mol. Struct. 2014, 1058, 114-121.

(25) Madusanka, N.; Eddleston, M. D.; Arhangelskis, M.; Jones, W. Polymorphs, Hydrates and Solvates of a Co-Crystal Ofcaffeine with Anthranilic Acid. Cryst. Eng. 2014, 70 (1), 72-80.

(26) FriŠčić, T.; Jones, W. Cocrystal Architecture and Properties: Design and Building of Chiral and Racemic Structures by Solid-solid Reactions. Faraday Discuss. 2007, 136, 167-178.

(27) Medina, C.; Daurio, D.; Nagapudi, K.; Alvarez-Núñez, F. Manufacture of Pharmaceutical Co-Crystals Using Twin Screw Extrusion: A Solvent-Less and Scalable Process. J. Pharm. Sci. 2010, 99 (4), 1693-1696.

(28) Dhumal, R.; Kelly, A.; York, P.; Coates, P.; Paradkar, A. Cocrystalization and Simultaneous Agglomeration Using Hot Melt Extrusion. Pharm. Res. 2010, 27 (12), 2725-2733.

(29) Daurio, D.; Medina, C.; Saw, R.; Nagapudi, K.; Alvarez-Núñez, F. Application of Twin Screw Extrusion in the Manufacture of Cocrystals, Part I: Four Case Studies. Pharmaceutics 2011, 3, 582-600.

(30) Kelly, A. L.; Gough, T.; Dhumal, R. S.; Halsey, S. A.; Paradkar, A. Monitoring Ibuprofen-nicotinamide Cocrystal Formation during Solvent Free Continuous Cocrystallization (SFCC) Using near Infrared Spectroscopy as a PAT Tool. Int. J. Pharm. 2012, 426 (1-2), 15-20.

(31) Kulkarni, C.; Wood, C.; Kelly, A. L.; Gough, T.; Blagden, N.; Paradkar, A. Stoichiometric Control of Co-Crystal Formation by Solvent Free Continuous 
(32) Remenar, J. F.; Peterson, M. L.; Stephens, P. W.; Zhang, Z.; Zimenkov, Y.; Hickey, M. B. Celecoxib:Nicotinamide Dissociation: Using Excipients To Capture the Cocrystal's Potential. Mol. Pharm. 2007, 4 (3), 386-400.

(33) Etter, M. C.; Reutzel, S. M.; Choo, C. G. Self-Organization of Adenine and Thymine in the Solid State. J. Am. Chem. Soc. 1993, 115 (10), 4411-4412.

(34) Grzesiak, A. L.; Uribe, F. J.; Ockwig, N. W.; Yaghi, O. M.; Matzger, A. J. Polymer-Induced Heteronucleation for the Discovery of New Extended Solids. Angew. Chemie - Int. Ed. 2006, 45 (16), 2553-2556.

(35) Hasa, D.; Schneider Rauber, G.; Voinovich, D.; Jones, W. Cocrystal Formation through Mechanochemistry: From Neat and Liquid-Assisted Grinding to Polymer-Assisted Grinding. Angew. Chemie 2015, 127 (25), 7479-7483.

(36) Qiu, S.; Li, M. Effects of Coformers on Phase Transformation and Release Profiles of Carbamazepine Cocrystals in Hydroxypropyl Methylcellulose Based Matrix Tablets. Int. J. Pharm. 2015, 479 (1), 118-128.

(37) Yazdanian, M.; Briggs, K.; Jankovsky, C.; Hawi, A. The "high Solubility" definition of the Current FDA Guidance on Biopharmaceutical Classification System May Be Too Strict for Acidic Drugs. Pharm. Res. 2004, 21 (2), 293299.

(38) Alshahateet, S. F. Synthesis and Supramolecularity of Hydrogen-Bonded Cocrystals of Pharmaceutical Model Rac-Ibuprofen with Pyridine Derivatives. Mol. Cryst. Liq. Cryst. 2010, 533 (1), 152-161.

(39) Aakeröy, C. B.; Beatty, A. M.; Helfrich, B. A. A High-Yielding Supramolecular Reaction. J. Am. Chem. Soc. 2002, 124 (48), 14425-14432.

(40) Aakeröy, C. B.; Beatty, A. M.; Helfrich, B. A.; Nieuwenhuyzen, M. Do Polymorphic Compounds Make Good Cocrystallizing Agents? A Structural Case Study That Demonstrates the Importance of Synthon Flexibility. Cryst. Growth Des. 2003, 3 (2), 159-165.

(41) Bathori, N. B.; Lemmerer, A.; Venter, G. A.; Bourne, S. A.; Caira, M. R. Pharmaceutical Co-Crystals with Isonicotinamide-Vitamin B3, Clofibric Acid, and Diclofenac-and Two Isonicotinamide Hydrates. Cryst. Growth Des. 2011, 11, 75-87.

(42) Thommes, M.; Ely, D. R.; Carvajal, M. T.; Pinal, R. Improvement of the Dissolution Rate of Poorly Soluble Drugs by Solid Crystal Suspensions. Mol. Pharm. 2011, 8, 727-735.

(43) Padrela, L.; de Azevedo, E. G.; Velaga, S. P. Powder X-Ray Diffraction Method for the Quantification of Cocrystals in the Crystallization Mixture . Drug Dev. Ind. Pharm. 2012, 38 (8), 923-929.

(44) Guidelines, I. C. H. Internation Conference on Harmonization (ICH) Harmonized Tripartite Guideline, Note for Guidance on Validation of Analytical Procedures: Methodology. ICH Steering Committee 1996.

(45) Leiserowits, L. Molecular Packing Modes. Carboxylic Acids. Acta Crystallogr. Sect. B Struct. Crystallogr. Cryst. Chem. 1976, 32, 775-802.

(46) Desiraju, G. R. Crystal Engineering: The Design of Organic Solids; Elsevier: Amsterdam, 1989.

(47) Steiner, T. The Hydrogen Bond in the Solid State. Angew. Chemie Int. Ed. 2002, 41 (1), 48-76.

(48) Oswald, I. D. H.; Motherwell, W. D. S.; Parsons, S. A 1 : 2 Co-Crystal of Isonicotinamide and Propionic Acid . Acta Crystallogr. Sect. E Struct. Reports Online 2004, 60 (12). 
(49) Vueba, M. L.; Pina, M. E.; Batista De Carvalho, L. A. E. Conformational Stability of Ibuprofen: Assessed by DFT Calculations and Optical Vibrational Spectroscopy. J. Pharm. Sci. 2008, 97 (2), 845-859.

(50) Silverstein, M.; Bassler, G. C.; Morrill, T. C. Spectrometric Identification of Organic Compounds ; J. Wiley \& Son Inc.: New York, 1991; Vol. 5th, pp 117-123.

(51) Logansen, A. V; Kurkchi, G. A.; Dement'eva, L. A. Infrared Spectra of Primary Amides in the vNH Range. J. Struct. Chem. 1977, 18 (4), 589-595.

(52) Sharma, B. K. Infrared Spectroscopy. In Spectroscopy; GOEL Publishing House: Meerut Delhi, 2007; Vol. 20th, pp 309-311.

(53) Filho, O. T.; Pinheiro, J. C.; da Costa, E. B.; Kondo, R. T.; de Souza, R. A.; Nogueira, V. M.; Mauro, A. E. Theoretical and Experimental Study of the Infrared Spectrum of Isonicotinamide. J. Mol. Struct. THEOCHEM 2006, 763, 175-179.

(54) Socrates, G. Infrared and Raman Characteristic Group Frequencies; 2004.

(55) Ramalingam, S.; Periandy, S.; Govindarajan, M.; Mohan, S. FT-IR and FTRaman Vibrational Spectra and Molecular Structure Investigation of Nicotinamide: A Combined Experimental and Theoretical Study. Spectrochim. Acta. A. Mol. Biomol. Spectrosc. 2010, 75 (5), 1552-1558.

(56) Tothadi, S.; Desiraju, G. R. Unusual Co-Crystal of Isonicotinamide: The Structural Landscape in Crystal Engineering. Philos. Trans. R. Soc. A Math. Phys. Eng. Sci. 2012, 370, 2900-2915.

(57) Steiner, T.; Desiraju, G. R. The Weak Hydrogen Bond in Structural Chemistry and Biology; Oxford University Press: New York, 1999.

(58) Doherty, C.; York, P. Fresemide Crystal Forms; Solid State and Physicochemical Analyses. Int. J. Pharm. 1988, 47 (1-3), 141-155.

(59) Romero, A. J.; Savastano, L.; Rhodes, C. T. Monitoring Crystal Modifications in Systems Containing Ibuprofen. Int. J. Pharm. 1993, 99 (2-3), 125-134.

(60) Lu, E.; Rodriguez-Hornedo, N.; Suryanarayanan, R. A Rapid Thermal Method for Cocrystal Screening. Cryst. Eng. Commun. 2008, 10, 665-668.

(61) FriŠčić, T.; Jones, W. Benefits of Cocrystallisation in Pharmaceutical Materials Science: An Update. J. Pharm. Pharmacol. 2010, 62, 1547-1559.

(62) Schultheiss, N.; Newman, A. W. Pharmaceutical Cocrystals and Their Physicochemical Properties. Cryst. Growth Des. 2009, 9 (6), 2950-2967.

(63) Almarsson, Ö.; Peterson, M. L.; Zaworotko, M. J. The A to Z of Pharmaceutical Cocrystals: A Decade of Fast Moving New Science and Patents. Pharm. Pat. Anal. 2012, 1 (3), 313-327.

(64) Alhalaweh, A.; Velaga, S. P. Formation of Cocrystals from Stoichiometric Solutions of Incongruently Saturating Systems by Spray Drying. Cryst. Growth Des. 2010, 10 (8), 3302-3305.

(65) Alhalaweh, A.; Kaialy, W.; Buckton, G.; Gill, H.; Nokhodchi, A.; Velaga, S. P. Theophylline Cocrystals Prepared by Spray Drying: Physicochemical Properties and Aerosolization Performance. AAPS PharmSciTech 2013, 14 (1), 265-276.

(66) Gryczke, A.; Schminke, S.; Maniruzzaman, M.; Beck, J.; Douroumis, D. Development and Evaluation of Orally Disintegrating Tablets (ODTs) Containing Ibuprofen Granules Prepared by Hot Melt Extrusion. Colloids Surfaces B Biointerfaces 2011, 86 (2), 275-284.

(67) Quentiros, D. A.; Allemandi, D. A.; Manzo, R. H. Equilibrium and Release Properties of Aqueous Dispersions of Non-Steroidal Anti-Inflammatory Drugs 
Complexed with Polyelectrolyte Eudragit E 100. Sci. Pharm. 2012, 80 (2), 487-496.

(68) Onoue, S.; Kojo, Y.; Aoki, Y.; Kawabata, Y.; Yamauchi, Y.; Yamada, S. Physical Chemical and Pharmacokinetic Characterization of Amorphous Solid Dispersion of Tranilast with Enhanced Solubility in Gastric Fluid and Improved Oral Bioavailability. Drug Metab. Pharmacokinet. Adv. Publ. 2012.

(69) Acton, Q. A. Sugar Alcohols-Advances in Research and Application; ScholarlyEditions: Atlanta, Geogia, 2013; Vol. 2013.

(70) Aakeröy, C. B.; Grommet, A. B.; Desper, J. Co-Crystal Screening of Diclofenac. Pharmaceutics 2011, 3 (3), 601-614.

(71) Alatas, F.; Soewandhi, S. N.; Sasongko, L.; Ismunandar; Uekusa, H. Cocrystal Formation between Didanosine and Two Aromatic Acids. Int. J. Pharm. Pharm. Sci. 2013, 5 (Suppl 3), 275-280.

(72) Allen, L. V. J.; Yanchick, V. A.; Maness, D. D. Dissolution Rates of Corticosteroids Utilizing Sugar Glass Dispersions . J. Pharm. Sci. 1977, 66 (4), 494-497.

(73) Leuner, C.; Dressman, J. Improving Drug Solubility for Oral Delivery Using Solid Dispersions. Eur. J. Pharm. Biopharm. 2000, 50 (1), 47-60.

(74) Perissutti, B.; Newton, J. M.; Podczeck, F.; Rubessa, F. Preparation of Extruded Carbamazepine and PEG 4000 as a Potential Rapid Release Dosage Form. Eur. J. Pharm. Biopharm. 2002, 53 (1), 125-132.

(75) Li, C.; Li, C.; Le, Y.; Chen, J.-F. Formation of Bicalutamide Nanodispersion for Dissolution Rate Enhancement. Int. J. Pharm. 2011, 404 (1-2), 257-263.

(76) Eddleston, M. D.; Patel, B.; Day, G. M.; Jones, W. Cocrystallization by FreezeDrying: Preparation of Novel Multicomponent Crystal Forms. Cryst. Growth Des. 2013, 13 (10), 4599-4606.

(77) Rumondor, A. C. F.; Stanford, L. A.; Taylor, L. S. Effects of Polymer Type and Storage Relative Humidity on the Kinetics of Felodipine Crystallization from Amorphous Solid Dispersions. Pharm. Res. 2009, 26 (12), 2599-2606.

(78) Stevens, M. J.; Covas, J. A. Extruder Principles and Operation; Chapman \& Hall: 2-6 Boundary Row, London SE1 8HN, UK, 1995; Vol. Second edi.

(79) Dreiblatt, A. Process Design. In Pharmaceutica Extrusion Technology; Ghebre-Sellassie, I., Martin, C., Eds.; Marcel Dekker, Inc.: New York, 2003; pp 153-169.

(80) Thiele, W. Twin-Screw Extrusion and Screw Design. In Pharmaceutical Extrusion Technology; Ghebre-Sellassie, I., Martin, C., Eds.; Marcel Dekker, Inc: New York, 2003; pp 69-98.

(81) Douroumis, D. Hot-Melt Extrusion: Pharmaceutical Extrusions; John Wiley \& Sons Ltd: West Sussex, UK, 2012.

(82) Reitz, E.; Podhaisky, H.; Ely, D.; Thommes, M. Residence Time Modeling of Hot Melt Extrusion Processes. Eur. J. Pharm. Biopharm. 2013, 85 (3 PART B), 1200-1205. 
